\title{
Commutators of $\theta$-type generalized fractional integrals on non-homogeneous spaces
}

\section{Guanghui Lu ${ }^{1 *}$}

\section{"Correspondence:}

Ighwmm1989@126.com

${ }^{1}$ College of Mathematics and

Statistics, Northwest Normal

University, Lanzhou, Gansu 730070,

P.R. China

\section{勿 Springer}

\begin{abstract}
The aim of this paper is to establish the boundednes of the commutator $\left[b, T_{\alpha}\right]$ generated by $\theta$-type generalized fractional integral $T_{\alpha}$ and $b \in \widetilde{\operatorname{RBMO}}(\mu)$ over a non-homogeneous metric measure space. Under the assumption that the dominating function $\lambda$ satisfies the $\epsilon$-weak reverse doubling condition, the author proves that the commutator $\left[b, T_{\alpha}\right]$ is bounded from the Lebesgue space $L^{p}(\mu)$ into the space $L^{q}(\mu)$ for $\frac{1}{q}=\frac{1}{p}-\alpha$ and $\alpha \in(0,1)$, and bounded from the atomic Hardy space $\widetilde{H}_{b}^{1}(\mu)$ with discrete coefficient into the space $L \frac{1}{1-\alpha}, \infty(\mu)$. Furthermore, the boundedness of the commutator $\left[b, T_{\alpha}\right]$ on a generalized Morrey space and a Morrey space is also got.
\end{abstract}

MSC: 42B35; 47B47; 30L99

Keywords: Non-homogeneous metric measure space; $\theta$-type generalized fractional integral; Commutator; $\widetilde{\operatorname{RBMO}}(\mu)$; Hardy space $\widetilde{H}_{b}^{1}(\mu)$

\section{Introduction}

The theories of the function spaces and the singular integral operators play an important role in the fields of the harmonic analysis and PDE. In particular, during the past 20 to 25 years, many authors have paid much attention to the space of homogeneous type in the sense of Coifman and Weiss $[4,5]$ and the metric measure space endowed with nondoubling measure; for example, see $[8,15,17,18,20-22]$ and the references therein.

However, the non-doubling measure may not satisfy the well-known doubling condition being a key assumption on spaces of homogeneous type. To solve this problem, Hytönen in [9] introduced a new class of metric measure spaces satisfying the so-called upper doubling and the geometrically doubling conditions (see Definitions 1.1 and 1.3 below, respectively). For convenience, the new metric measure space is now called a non-homogeneous metric measure space. Since then, many papers about the different kinds of function spaces and singular integral operators on non-homogeneous metric measure space have been widely focused on; for example, see $[2,7,11-14,16,19]$ and the references therein. Furthermore, see the monograph [23] to find the more development on harmonic analysis in this new context.

(c) The Author(s) 2020. This article is licensed under a Creative Commons Attribution 4.0 International License, which permits use sharing, adaptation, distribution and reproduction in any medium or format, as long as you give appropriate credit to the original author(s) and the source, provide a link to the Creative Commons licence, and indicate if changes were made. The images or other third party material in this article are included in the article's Creative Commons licence, unless indicated otherwise in a credit line to the material. If material is not included in the article's Creative Commons licence and your intended use is not permitted by statutory regulation or exceeds the permitted use, you will need to obtain permission directly from the copyright holder. To view a copy of this licence, visit http://creativecommons.org/licenses/by/4.0/. 
Let $(\mathcal{X}, d, \mu)$ be a non-homogeneous metric measure space in the sense of Hytönen [9]. In this setting, Lin et al. [12] proved that the commutator $T_{b}:=b T-T b$ generated by the Calderón-Zygmund operator $T$ and the function $b \in \widehat{\operatorname{RBMO}}(\mu)$ is bounded from the atomic Hardy space $\widetilde{H}^{1}(\mu)$ with discrete coefficient into the space $L^{1, \infty}(\mu)$, and bounded from Lebesgue space $L^{p}(\mu)$ into the space $L^{p}(\mu)$ for $p \in(1, \infty)$. Moreover, Ri and Zhang in [16] proved that the commutators of $\theta$-type Calderón-Zygmund operators with RBMO functions is bounded from the $L^{\infty}(\mu)$ into the space $\operatorname{RBMO}(\mu)$, and bounded from the Hardy space $H^{1}(\mu)$ into the $L^{1}(\mu)$. In [7], Fu et al. established some equivalent characterizations for the boundedness of the generalized fractional integrals over $(\mathcal{X}, d, \mu)$, moreover, the boundedness of the multilinear commutators of generalized fractional integrals with $\operatorname{RBMO}(\mu)$ functions on Orlicz spaces is obtained. Motivated by these results, in this paper, we will mainly establish the boundedness of the commutator $\left[b, T_{\alpha}\right]$ generated by $\theta$-type generalized fractional integral $T_{\alpha}$ and $b \in \widehat{\mathrm{RBMO}}(\mu)$ on the Lebesgue space, atomic Hardy space with discrete coefficient, Morrey space and generalized Morrey space.

Before presenting the organization of this paper, we need to recall some necessary notions. The following definitions of the upper doubling and geometrically doubling conditions are from [9].

Definition 1.1 ([9]) A metric measure space $(\mathcal{X}, d, \mu)$ is said to be upper doubling, if $\mu$ is Borel measure on $\mathcal{X}$ and there exist a dominating function

$$
\lambda: \mathcal{X} \times(0, \infty) \rightarrow(0, \infty)
$$

and a positive constant $C_{\lambda}$ depending only on $\lambda$ such that, for each $x \in \mathcal{X}, r \rightarrow \lambda(x, r)$ is non-decreasing and, for all $x \in \mathcal{X}$ and $r \in(0, \infty)$,

$$
\mu(B(x, r)) \leq \lambda(x, r) \leq C_{\lambda} \lambda\left(x, \frac{r}{2}\right)
$$

Remark 1.2 From [10], Hytönen et al. have showed that there exists another dominating function $\tilde{\lambda}$ such that $\tilde{\lambda} \leq \lambda, C_{\tilde{\lambda}} \leq C_{\lambda}$ and, for all $x, y \in \mathcal{X}$ with $d(x, y) \leq r$,

$$
\tilde{\lambda}(x, r) \leq C_{\tilde{\lambda}} \tilde{\lambda}(y, r) .
$$

Thus, we always assume that the dominating function $\lambda$ as in (1.1) satisfies (1.2) in this paper.

Definition 1.3 ([9]) A metric space $(\mathcal{X}, d)$ is said to be geometrically doubling, if there exists some $N_{0} \in \mathbb{N}$ such that, for any ball $B(x, r) \subset \mathcal{X}$, there exists a finite ball covering $\left\{B\left(x_{i}, \frac{r}{2}\right)\right\}_{i}$ of $B(x, r)$ such that the cardinality of this covering is at most $N_{0}$.

Remark 1.4 Hytönen in [9] has showed that, if a metric space $(\mathcal{X}, d)$ is geometrically doubling, then, for any $\epsilon \in(0,1)$ and ball $B(x, r) \subset \mathcal{X}$, there exists a finite ball covering $\left\{B\left(x_{i}, \epsilon r\right)\right\}_{i}$ of $B(x, r)$ such that the cardinality of this covering is at most $N_{0} \epsilon^{-n}$. Here and in what follows, $N_{0}$ is as in Definition 1.3 and $n:=\log _{2} N_{0}$.

We now recall the definition of the discrete coefficient $\widetilde{K}_{B, S}^{(\rho)}$ originally introduced by Bui and Duong (see [1]), which is closer to the quantity introduced by Tolsa in [20]. 
Definition 1.5 ([1]) For any $\rho \in(1, \infty)$ and any two balls $B \subset S \subset \mathcal{X}$, let

$$
\widetilde{K}_{B, S}^{(\rho)}:=1+\sum_{\left.k=-\left\lfloor\log _{\rho}\right\rfloor\right\rfloor}^{N_{B, S}^{(\rho)}} \frac{\mu\left(\rho^{k} B\right)}{\lambda\left(c_{B}, \rho^{k} r_{B}\right)},
$$

where $N_{B, S}^{(\rho)}$ represents the smallest integer satisfying $\rho^{N_{B, S}^{(\rho)}} r_{B} \geq r_{S}$, and $c_{B}$ and $r_{B}$ are the center and radius of ball $B$, respectively.

Remark 1.6 A continuous version of the coefficient $\widetilde{K}_{B, S}^{(\rho)}$ was given in [9] and [10] as follows. That is, for any two balls $B \subset S \subset \mathcal{X}$, define

$$
K_{B, S}:=1+\int_{(2 S) \backslash B} \frac{\mathrm{d} \mu(x)}{\lambda\left(c_{B}, d\left(x, c_{B}\right)\right)} .
$$

In general, $K_{B, S}$ and $\widetilde{K}_{B, S}^{(\rho)}$ are not equivalent, but, if we take $(\mathcal{X}, d, \mu)=\left(\mathbb{R}^{d},|\cdot|, \mu\right)$ and $\lambda(x, r):=C r^{d}$ as in (1.1), it is not difficult to find that

$$
K_{B, S} \sim \widetilde{K}_{B, S}^{(\rho)}
$$

with implicit equivalent positive constants independent of the balls $B$ and $S$; see [12] for more details. In addition, by (1.1) and a change of variables, it is easy to obtain the other form of the $\widetilde{K}_{B, S}^{(\rho)}$, that is,

$$
\widetilde{K}_{B, S}^{(\rho)} \sim 1+\sum_{k=1}^{N_{B, S}^{(\rho)}\left\lfloor\left\lfloor\log _{\rho} 2\right\rfloor+1\right.} \frac{\mu\left(\rho^{k} B\right)}{\lambda\left(c_{B}, \rho^{k} r_{B}\right)} .
$$

Next, we recall the following definition of the fractional coefficient $\widetilde{K}_{B, S}^{\alpha}$ given in [7].

Definition 1.7 ([7]) Let $\alpha \in[0,1)$. For any two balls $B:=B\left(c_{B}, r_{B}\right) \subset S, \widetilde{K}_{B, S}^{\alpha}$ is defined by

$$
\widetilde{K}_{B, S}^{\alpha}:=1+\sum_{k=1}^{N_{B, S}}\left[\frac{\mu\left(6^{k} B\right)}{\lambda\left(c_{B}, 6^{k} r_{B}\right)}\right]^{1-\alpha}
$$

where $N_{B, S}$ is the smallest integer satisfying $6^{N_{B, S}} r_{B} \geq r_{S}$.

Remark 1.8 If we take $\alpha \equiv 0$ in Definition 1.7, then the fractional coefficient $\widetilde{K}_{B, S}^{\alpha}$ is just the $\widetilde{K}_{B, S}$ introduced by Bui and Duong in [1]. Moreover, the reader can see [7, Lemma 3.4] to find the other properties of the coefficient $\widetilde{K}_{B, S}$.

Although the measure doubling condition is not assumed uniformly for all balls on $(\mathcal{X}, d, \mu)$, Hytönen has showed that there are many balls having $(\eta, \beta)$-doubling property. Namely, for $\eta, \beta>1$, a ball $B \subset \mathcal{X}$ is said to be $(\eta, \beta)$-doubling if $\mu(\eta B) \leq \beta \mu(B)$. Meanwhile, Hytönen [9] proved that if a metric measure space $(\mathcal{X}, d, \mu)$ is upper doubling and $\beta>C_{\lambda}^{\log _{2} \eta}=: \eta^{v}$, then, for each ball $B \subset \mathcal{X}$, there exists some $j \in \mathbb{Z}_{+}$such that $\eta^{j} B$ is $(\eta, \beta)$ doubling. Moreover, let $(\mathcal{X}, d)$ be a geometrically doubling, $\beta>\eta^{n}$ with $n:=\log _{2} N_{0}$ and $\mu$ 
Borel measure on $\mathcal{X}$ being finite on bounded sets. Hytönen also showed that there exist arbitrarily small $(\eta, \beta)$-doubling balls centered at $x$ for $\mu$-a.e $x \in \mathcal{X}$. Furthermore, the radius of there balls may be chosen to be of the form $\eta^{-j} r$ for $j \in \mathbb{N}$ and any preassigned number $r \in(0, \infty)$. Throughout this paper, for any $\eta \in(1, \infty)$ and ball $B$, the smallest $\left(\eta, \beta_{\eta}\right)$ doubling ball of the form $\eta^{j} B$ with $j \in \mathbb{Z}_{+}$is denoted by $\tilde{B}^{\eta}$, where

$$
\beta_{\eta}:=\max \left\{\eta^{3 n}, \eta^{3 \nu}\right\}+30^{n}+30^{\nu}
$$

In this paper, if there is no special explanation, we always set $\eta=6$ and simply denote $\widetilde{B}^{6}$ by $\widetilde{B}$.

The definition of $\theta$-type generalized fractional integral is as follows.

Definition 1.9 Let $\alpha \in(0,1)$, and $\theta$ be a non-negative and non-decreasing function on $(0, \infty)$ satisfying the condition

$$
\int_{0}^{1} \frac{\theta(t)}{t} \mathrm{~d} t<\infty
$$

A kernel $K_{\alpha}(\cdot, \cdot) \in L_{\text {loc }}^{1}(\mathcal{X} \times \mathcal{X} \backslash\{(x, x): x \in \mathcal{X}\})$ is called a $\theta$-type generalized fractional integral kernel if there exists a constant $C_{K_{\alpha}}>0$ only depending on $K_{\alpha}$, such that

$$
\left|K_{\alpha}(x, y)\right| \leq \frac{C_{K_{\alpha}}}{[\lambda(x, d(x, y))]^{1-\alpha}}
$$

for all $x, y \in \mathcal{X}$ with $x \neq y$, and for all $x, x^{\prime}, y \in \mathcal{X}$,

$$
\begin{aligned}
& \left|K_{\alpha}(x, y)-K_{\alpha}\left(x^{\prime}, y\right)\right|+\left|K_{\alpha}(y, x)-K_{\alpha}\left(y^{\prime}, x\right)\right| \\
& \quad \leq C_{K_{\alpha}} \theta\left(\frac{d(x, x)}{d(x, y)}\right) \frac{1}{[\lambda(x, d(x, y))]^{1-\alpha}},
\end{aligned}
$$

provided $c_{K_{\alpha}} d\left(x, x^{\prime}\right) \leq d(x, y)$ with $c_{K_{\alpha}} \in(0, \infty)$.

Moreover, if we take $\theta(t)=t^{\delta}$ with $\delta \in(0,1]$ in (1.10), then the $\theta$-type generalized fractional integral kernel is just the generalized fractional integral (see [7]).

Let $L_{b}^{\infty}(\mu)$ be the space of all $L^{\infty}(\mu)$ functions with bounded support. A linear operator $T_{\alpha}$ is called a $\theta$-type generalized fractional integral with kernel $K_{\alpha}$ satisfying (1.9) and (1.10) if, for all $f \in L_{b}^{\infty}(\mu)$ and $x \notin \operatorname{supp}(f)$,

$$
T_{\alpha} f(x):=\int_{\mathcal{X}} K_{\alpha}(x, y) f(y) \mathrm{d} \mu(y) .
$$

We now recall the notation of the space $\widetilde{\operatorname{RBMO}}(\mu)$ given in [6].

Definition 1.10 ([6]) Let $\rho \in(1, \infty)$ and $\gamma \in[1, \infty)$. A function $f \in L_{\text {loc }}^{1}(\mu)$ is said to be in the space $\widetilde{\operatorname{RBMO}}_{\rho, \gamma}(\mu)$ if there exist a positive constant $C$ and, for any ball $B \subset \mathcal{X}$, a number $f_{B}$ such that

$$
\frac{1}{\mu(\rho B)} \int_{B}\left|f(x)-f_{B}\right| \mathrm{d} \mu(x) \leq C
$$


and, for any two balls $B$ and $S$ such that $B \subset S$,

$$
\left|f_{B}-f_{S}\right| \leq C\left[\widetilde{K}_{B, S}^{(\rho)}\right]^{\gamma}
$$

The infimum of the positive constant $C$ satisfying both (1.12) and (1.13) is defined to be the $\widetilde{\operatorname{RBMO}}_{\rho, \gamma}(\mu)$ norm of $f$ and denoted by $\|f\|_{\widehat{\operatorname{BBMO}}_{\rho, \gamma}(\mu)}$.

From [6], Fu et al. have showed that the space $\widetilde{\operatorname{RBMO}_{\rho, \gamma}}(\mu)$ is independent of the choices of $\rho \in(1, \infty)$ and $\gamma \in[1, \infty)$. Thus, the space $\widetilde{\operatorname{RBMO}}_{\rho, \gamma}(\mu)$ is simply denoted by $\widetilde{\operatorname{RBMO}}(\mu)$. Moreover, we can see [13, Remark 2.14] to find more properties of the space $\widetilde{\operatorname{RBMO}}(\mu)$.

Given a function $b \in \widehat{\operatorname{RBMO}}(\mu)$, the commutator $\left[b, T_{\alpha}\right]$ associated with the $\theta$-type generalized fractional integral $T_{\alpha}$ is, respectively, defined by

$$
\left[b, T_{\alpha}\right] f(x)=\int_{\mathcal{X}} K_{\alpha}(x, y)[b(x)-b(y)] f(y) \mathrm{d} \mu(y) .
$$

The following notion of the $\epsilon$-weak reverse doubling condition is from [7]; also see [13].

Definition 1.11 ([7]) Let $\epsilon \in(0, \infty)$. A dominating function $\lambda$ is said to satisfy the $\epsilon$-weak reverse doubling condition if, for all $r \in(0,2 \operatorname{diam}(\mathcal{X}))$ and $a \in(1,2 \operatorname{diam}(\mathcal{X}) / r)$, there exists a number $C(a) \in[1, \infty)$, depending only on $a$ and $\mathcal{X}$, such that, for all $x \in \mathcal{X}$,

$$
\lambda(x, a r) \geq C(a) \lambda(x, r)
$$

and, moreover,

$$
\sum_{k=1}^{\infty} \frac{1}{\left[C\left(a^{k}\right)\right]^{\epsilon}}<\infty .
$$

The organization of this paper is as follows. In Sect. 2, we mainly recall some necessary lemmas being used in the proof of the main theorems. In Sect. 3, we will prove that the commutator $\left[b, T_{\alpha}\right]$ generated by the $\theta$-type fractional integral operator $T_{\alpha}$ and $b \in \widetilde{\operatorname{RBMO}}(\mu)$ is bounded from the Lebesgue space $L^{p}(\mu)$ into the space $L^{q}(\mu)$, where $\frac{1}{q}=\frac{1}{p}-\alpha$ with $\alpha \in(0,1)$ and $p \in\left(1, \frac{1}{\alpha}\right)$. In Sect. 4 , via decomposition of the atomic, the boundedness of the commutator $\left[b, T_{\alpha}\right]$ from the atomic Hardy space $\widetilde{H}_{b}^{1}(\mu)$ with discrete coefficient into the space $L^{\frac{1}{1-\alpha}, \infty}(\mu)$ is obtained. The boundedness of the commutator $\left[b, T_{\alpha}\right]$ on the Morrey space and the generalized Morrey space is also presented in Sects. 5 and 6 , respectively.

Finally, we make some conventions on notation. Throughout the paper, $C$ represents for a positive constant that is independent of the main parameters involved, but may be different from line to line. For a $\mu$-measurable set $E, \chi_{E}$ denotes its characteristic function. For any $p \in[1, \infty]$, we denote by $p^{\prime}$ its conjugate index, that is, $\frac{1}{p}+\frac{1}{p^{\prime}}=1$. In addition, for any $f \in L_{\mathrm{loc}}^{1}(\mu)$ and any measurable set $E$ of $\mathcal{X}, m_{B}(f)$ represents the mean value of the function $f$ over ball $B$, namely, $m_{B}(f):=\frac{1}{\mu(B)} \int_{B} f(x) \mathrm{d} \mu(x)$. 


\section{Preliminaries}

In this section, we will recall some necessary lemmas which is used in the proof of the main theorems in this paper. We first need to recall some properties of the discrete coefficient $\widetilde{K}_{B, S}^{(\rho)}($ see [12]).

Lemma 2.1 ([13]) Let $(\mathcal{X}, d, \mu)$ be a non-homogeneous metric measure space.

(1) For any $\rho \in(1, \infty)$, there exists a positive constant $C_{(\rho)}$, depending on $\rho$, such that, for any balls $B \subset R \subset S, \widetilde{K}_{B, R}^{(\rho)} \leq C_{(\rho)} \widetilde{K}_{B, S}^{(\rho)}$.

(2) For any $\alpha \in[1, \infty)$ and $\rho \in(1, \infty)$, there exists a positive constant $C_{(\alpha, \rho)}$, depending on $\alpha$ and $\rho$, such that, for all balls $B \subset S$ with $r_{S} \leq \alpha r_{B}, \widetilde{K}_{B, S}^{(\rho)} \leq C_{(\alpha, \rho)}$.

(3) For any $\rho \in(1, \infty)$, there exists a constant $C_{(\rho, v)}>0$, depending on $\rho$ and $v$, such that, for all balls $B, \widetilde{K}_{B, \widetilde{B}^{\rho}}^{(\rho)} \leq C_{(\rho, v)}$. Moreover, letting $\alpha, \beta \in(1, \infty), B \subset S$ be any two concentric balls such that there exists no $(\alpha, \beta)$-doubling ball in the form of $\alpha^{k} B$ with $k \in \mathbb{N}$, satisfying $B \subset \alpha^{k} B \subset S$, then there exists a positive constant $C_{(\alpha, \beta, v)}$, depending on $\alpha, \beta$ and $\nu$, such that $\widetilde{K}_{B, S}^{(\rho)} \leq C_{(\alpha, \beta, v)}$.

(4) For any $\rho \in(1, \infty)$, there exists a constant $c_{(\rho, v)}>0$, depending on $\rho$ and $v$, such that, for all balls $B \subset R \subset S$,

$$
\widetilde{K}_{B, S}^{(\rho)} \leq \widetilde{K}_{B, R}^{(\rho)}+c_{(\rho, v)} \widetilde{K}_{R, S}^{(\rho)}
$$

(5) For any $\rho \in(1, \infty)$, there exists a positive constant $\widetilde{c}_{(\rho, v)}$, depending on $\rho$ and $v$, such that, for all balls $B \subset R \subset S, \widetilde{K}_{R, S}^{(\rho)} \leq \widetilde{c}_{(\rho, v)} \widetilde{K}_{B, S}^{(\rho)}$.

We now recall the following characterizations of the space $\widetilde{\operatorname{RBMO}}(\mu)$ given in [12].

Lemma 2.2 ([13]) Let $\tau, \rho \in(1, \infty)$, and $\beta_{\rho}$ be as in (1.7). For $f \in L_{\mathrm{loc}}^{1}(\mu)$, the following statements are mutually equivalent:

(1) $f \in \widehat{\operatorname{RBMO}}(\mu)$;

(2) there exists a positive constant $C$ such that, for all balls $B$,

$$
\frac{1}{\mu(\tau B)} \int_{B}\left|f(x)-m_{\widetilde{B}^{\rho}}(f)\right| \mathrm{d} \mu(x) \leq C
$$

and, for all $\left(\rho, \beta_{\rho}\right)$-doubling balls $B \subset S$,

$$
\left|m_{B}(f)-m_{S}(f)\right| \leq C \widetilde{K}_{B, S}^{(\rho)}
$$

Moreover, the infimum of the above constant $C$ in (2) is equivalent to $\|f\|_{\overparen{\operatorname{BMO}}(\mu)}$.

The following conclusion is from [13, Corollary 2.17].

Corollary $2.3([13])$ For any $\rho \in(1, \infty)$ and $p \in[1, \infty)$, there exists a positive constant $C$, such that, for all $f \in \widehat{\mathrm{RBMO}}(\mu)$ and ball $B$,

$$
\left(\frac{1}{\mu(\rho B)} \int_{B}\left|f(x)-f_{B}\right|^{p} \mathrm{~d} \mu(x)\right)^{\frac{1}{p}} \leq C\|f\|_{\widetilde{\operatorname{RMO}}(\mu)} .
$$

Also, we need to recall some results given in [1]. 
Lemma 2.4 ([1]) Let $(\mathcal{X}, d, \mu)$ be a metric measure space of non-homogeneous type.

(1) Let $p \in(1, \infty), r \in(1, p)$ and $\zeta \in[5, \infty)$. The following maximal operators defined, respectively, by setting, for all $f \in L_{\mathrm{loc}}^{1}(\mu)$ and $x \in \mathcal{X}$,

$$
\begin{aligned}
& M_{r, \zeta} f(x):=\sup _{B \ni x}\left(\frac{1}{\mu(\zeta B)} \int_{B}|f(y)|^{r} \mathrm{~d} \mu(y)\right)^{\frac{1}{r}}, \\
& N f(x):=\sup _{B \ni x, B \text { doubling }} \frac{1}{\mu(B)} \int_{B}|f(y)| \mathrm{d} \mu(y),
\end{aligned}
$$

and

$$
M_{(\zeta)} f(x):=\sup _{B \ni x} \frac{1}{\mu(\zeta B)} \int_{B}|f(y)| \mathrm{d} \mu(y),
$$

are bounded on $L^{p}(\mu)$ and also bounded from $L^{1}(\mu)$ into $L^{1, \infty}(\mu)$.

(2) For all $f \in L_{\text {loc }}^{1}(\mu)$, it holds true that $|f(x)| \leq N f(x)$ for $\mu$-almost every $x \in \mathcal{X}$.

Next, we recall the following lemma from [7].

Lemma 2.5 ([7]) Let $\alpha \in(0,1), s \in\left(1, \frac{1}{\alpha}\right), \zeta \in[5, \infty), p \in\left(s, \frac{1}{\alpha}\right)$ and $\frac{1}{q}=\frac{1}{p}-\alpha$. Then there exists a positive constant $C$ such that, for all $f \in L^{p}(\mu)$,

$$
\left\|M_{s, \zeta}^{(\alpha)} f\right\|_{L^{q}(\mu)} \leq C\|f\|_{L^{p}(\mu)}
$$

where

$$
M_{s, \zeta}^{(\alpha)} f(x):=\sup _{B \ni x}\left\{\frac{1}{[\mu(\zeta B)]^{1-\alpha s}} \int_{B}|f(y)|^{s} \mathrm{~d} \mu(y)\right\}^{\frac{1}{s}}
$$

and the supremum is taken over all balls $B \ni x$.

Let $\alpha \in(0,1)$. For all $f \in L_{\mathrm{loc}}^{1}(\mu)$ and $x \in \mathcal{X}$, the sharp maximal function $M^{\sharp, \alpha}$ of $f$ is defined by

$$
\widetilde{M}^{\sharp, \alpha} f(x):=\sup _{B \ni x} \frac{1}{\mu(6 B)} \int_{B}\left|f(y)-m_{\widetilde{B}}(f)\right| \mathrm{d} \mu(y)+\sup _{x \in \Delta_{x}} \frac{\left|m_{B}(f)-m_{S}(f)\right|}{\widetilde{K}_{B, S}^{\alpha}} .
$$

where $\Delta_{x}:=\{(B, S): x \in B \subset S$ and $B, S$ are doubling balls $\}$.

We now recall the following lemma from [7].

Lemma 2.6 ([7]) Let $f \in L_{\text {loc }}^{1}(\mu)$ satisfying that $\int_{\mathcal{X}} f(x) \mathrm{d} \mu(x)=0$ when $\|\mu\|:=\mu(\mathcal{X})<\infty$. Assume that, for some $p \in(1, \infty), \inf \{1, N f\} \in L^{p}(\mu)$. Then there exists a constant $C>0$, independent off, such that $\|N f\|_{L^{p}(\mu)} \leq C\left\|M^{\sharp, \alpha} f\right\|_{L^{p}(\mu)}$.

Finally, we recall the following lemmas given in [7].

Lemma 2.7 ([7]) For any $\alpha \in[0,1)$, there exists some positive constant $P_{\alpha}$ (big enough), depending only on $C_{\lambda}$ as in (1.1) and $\alpha$, such that, if $m \in \mathbb{N}, B_{1} \subset B_{2} \subset \cdots \subset B_{m}$ are concentric balls with $\widetilde{K}_{B_{i}, B_{i+1}}^{\alpha}>P_{\alpha}$ for $i \in\{1,2, \ldots, m-1\}$, then there exists a positive constant $C$, depending only on $C_{\lambda}$ and $\alpha$, such that $\sum_{i=1}^{m-1} \widetilde{K}_{B_{i}, B_{i+1}}^{\alpha} \leq C \widetilde{K}_{B_{1}, B_{m}}^{\alpha}$. 
Lemma 2.8 ([7]) For any $\alpha \in[0,1)$, there exists some positive constant $\widetilde{P}_{\alpha}$ (large enough), depending on $C_{\lambda}, \beta_{6}$ as in (1.7) with $\eta=6$ and $\alpha$, such that, if $x \in \mathcal{X}$ is some fixed point and $\left\{f_{B}\right\}_{b \ni x}$ is a collection of numbers such that $\left|f_{B}-f_{S}\right| \leq \widetilde{K}_{B, S}^{\alpha} C_{x}$ for all doubling balls $B \subset S$ with $x \in B$ satisfying $\widetilde{K}_{B, S}^{\alpha} \leq \widetilde{P}_{\alpha}$, then there exists a positive constant $C$, depending on $C_{\lambda}$, $\beta_{6}, \alpha$ and $\widetilde{P}_{\alpha}$, such that $\left|f_{B}-f_{S}\right| \leq C \widetilde{K}_{B, S}^{\alpha} C_{x}$ for all doubling balls $B \subset S$ with $x \in B$, where $C_{x}$ is a positive constant, depending on $x$, and $C$ a positive constant depending only on $C_{\lambda}, \beta_{6}$ and $\alpha$.

\section{Boundedness of $\left[b, T_{\alpha}\right]$ on Lebesgue space}

In this section, we will establish the boundedness of the commutator $\left[b, T_{\alpha}\right]$ generated by the $\theta$-type generalized fractional integral $T_{\alpha}$ and the space $\widetilde{\mathrm{RBMO}}(\mu)$ on the Lebesgue space $L^{p}(\mu)$ for $p \in(1, \infty)$. Moreover, the endpoint boundedness of the commutator $\left[b, T_{\alpha}\right]$ is also obtained.

We now state the main theorems of this section as follows.

Theorem 3.1 Let $b \in \widetilde{\mathrm{RBMO}}(\mu), \alpha \in(0,1), p \in\left(1, \frac{1}{\alpha}\right)$ with $\frac{1}{q}=\frac{1}{p}-\alpha$, and $K_{\alpha}$ satisfy (1.9) and (1.10). Suppose that $T_{\alpha}$ is as in (1.11). Then there exists a constant $C>0$, such that, for all $f \in L^{p}(\mu)$,

$$
\left\|\left[b, T_{\alpha}\right](f)\right\|_{L^{q}(\mu)} \leq C\|b\|_{\widetilde{\operatorname{BMO}}(\mu)}\|f\|_{L^{p}(\mu)}
$$

To prove the main theorem, we need to establish the following lemma about the $\theta$-type generalized fractional integral $T_{\alpha}$.

Lemma 3.2 Let $\alpha \in(0,1), p \in\left(1, \frac{1}{\alpha}\right)$ with $\frac{1}{q}=\frac{1}{p}-\alpha$, and $K_{\alpha}$ satisfy (1.9). Then there exists a positive constant $C$, such that, for all $f \in L^{p}(\mu)$,

$$
\left\|T_{\alpha}(f)\right\|_{L^{q}(\mu)} \leq C\|f\|_{L^{p}(\mu)}
$$

Proof For any $x \in \mathcal{X}$, by applying (1.9) and (1.11), we can get

$$
\begin{aligned}
\left|T_{\alpha} f(x)\right| & \leq \int_{\mathcal{X}}\left|K_{\alpha}(x, y)\right||f(y)| \mathrm{d} \mu(y) \\
& \leq C \int_{\mathcal{X}} \frac{|f(y)|}{[\lambda(x, d(x, y))]^{1-\alpha}} \mathrm{d} \mu(y) \\
& \leq C I_{\alpha}(|f|)(x),
\end{aligned}
$$

where $I_{\alpha}$ represents the fractional integral operator defined by

$$
I_{\alpha} f(x):=\int_{\mathcal{X}} \frac{f(y)}{[\lambda(x, d(x, y))]^{1-\alpha}} \mathrm{d} \mu(y), \quad x \in \mathcal{X} .
$$

Furthermore, by the $\left(L^{p}(\mu), L^{q}(\mu)\right)$-boundedness of $I_{\alpha}$ (see [7]), it is difficult to obtain

$$
\left\|T_{\alpha} f\right\|_{L^{q}(\mu)} \leq C\|f\|_{L^{p}(\mu)} .
$$

Hence, the proof of Lemma 3.2 is completed. 
Now we give the proof of Theorem 3.1.

Proof of Theorem 3.1 In a slightly modified way similar to that used in the proof of Theorem 3.10 in [7], it is not difficult to prove that the case $\mu(\mathcal{X})=\infty$ holds. Thus, without loss of generality, we may assume $\mu(\mathcal{X})=\infty$. Assume that $p \in\left(1, \frac{1}{\alpha}\right)$. First, we claim that, for all $r \in(1, \infty)$ and $f \in L^{p}(\mu)$,

$$
\widetilde{M}^{\sharp, \alpha}\left(\left[b, T_{\alpha}\right] f\right)(x) \leq C\|b\|_{\overparen{\operatorname{RBMO}}(\mu)}\left\{M_{r, 5}^{(\alpha)} f(x)+M_{r, 6}\left(T_{\alpha} f\right)(x)+T_{\alpha}(|f|)(x)\right\} .
$$

Once (3.1) is obtained, taking $1<r<p<\frac{1}{\alpha}$, by applying Lemmas 2.4, 2.5 and 2.6, we can deduce that

$$
\begin{aligned}
\left\|\left[b, T_{\alpha}\right] f\right\|_{L^{q}(\mu)} & \leq\left\|N\left(\left[b, T_{\alpha}\right] f\right)\right\|_{L^{q}(\mu)} \leq C\left\|\tilde{M}^{\sharp, \alpha}\left(\left[b, T_{\alpha}\right] f\right)\right\|_{L^{q}(\mu)} \\
& \leq C\|b\|_{\widetilde{\operatorname{BMMO}}(\mu)}\left\{\left\|M_{r, 5}^{(\alpha)} f\right\|_{L^{q}(\mu)}+\left\|M_{r, 6}\left(T_{\alpha} f\right)\right\|_{L^{q}(\mu)}+\left\|T_{\alpha}(|f|)\right\|_{L^{q}(\mu)}\right\} \\
& \leq C\|b\|_{\widetilde{\operatorname{BMMO}}(\mu)}\|f\|_{L^{p}(\mu)},
\end{aligned}
$$

which is the desired consequence.

Thus, we need to show (3.1). By the definition of the sharp maximal operator $\widetilde{M}^{\sharp, \alpha}$ in (2.6), we should show that, for all $x$ and balls $B$ with $B \ni x$,

$$
\frac{1}{\mu(6 B)} \int_{B}\left|\left[b, T_{\alpha}\right] f(y)-h_{B}\right| \mathrm{d} \mu(y) \leq C\|b\|_{\overparen{\operatorname{BMMO}}(\mu)}\left\{M_{r, 5}^{(\alpha)} f(x)+M_{r, 6}\left(T_{\alpha} f\right)(x)\right\} .
$$

and, for all balls $B, S$ and $B \ni x$,

$$
\left|h_{B}-h_{S}\right| \leq C\|b\|_{\overparen{\operatorname{BMO}}(\mu)}\left\{M_{r, 5}^{(\alpha)} f(x)+T_{\alpha}(|f|)(x)\right\} \widetilde{K}_{B, S}^{(\rho)} \widetilde{K}_{B, S}^{\alpha}
$$

where

$$
h_{B}:=m_{B}\left(T_{\alpha}\left(\left[b-m_{B}(b)\right] f \chi_{\mathcal{X} \backslash \frac{6}{5} B}\right)\right)
$$

and

$$
h_{S}:=m_{S}\left(T_{\alpha}\left(\left[b-m_{S}(b)\right] f \chi_{\mathcal{X} \backslash \frac{6}{5} S}\right)\right) .
$$

With a slightly modified argument similar to that used in the estimates (3.6), (3.7) and (3.8) in [7, Theorem 3.9], it is not difficult to see that (3.2) holds, too. However, to estimate (3.2), we still need to estimate the difference $\left|T_{\alpha}\left(\left[b-b_{B}\right] f_{2}\right)-h_{B}\right|$ with $f_{2}=f \chi_{\mathcal{X} \backslash \frac{6}{5} B}$. So, for any $y_{1}, y_{2} \in B$, write

$$
\begin{aligned}
& \left|T_{\alpha}\left(\left[b-b_{B}\right] f_{2}\right)\left(y_{1}\right)-T_{\alpha}\left(\left[b-b_{B}\right] f_{2}\right)\left(y_{2}\right)\right| \\
& \quad \leq \int_{\mathcal{X} \backslash \frac{6}{5} B}\left|K_{\alpha}\left(y_{1}, z\right)-K_{\alpha}\left(y_{2}, z\right)\right|\left|b(z)-b_{B}\right||f(z)| \mathrm{d} \mu(z) \\
& \quad \leq \int_{\mathcal{X} \backslash 6 B}\left|K_{\alpha}\left(y_{1}, z\right)-K_{\alpha}\left(y_{2}, z\right)\right|\left|b(z)-b_{B}\right||f(z)| \mathrm{d} \mu(z)
\end{aligned}
$$




$$
\begin{aligned}
& +\int_{6 B \backslash \frac{6}{5} B}\left|K_{\alpha}\left(y_{1}, z\right)-K_{\alpha}\left(y_{2}, z\right)\right|\left|b(z)-b_{B}\right||f(z)| \mathrm{d} \mu(z) \\
= & : \mathrm{A}_{1}+\mathrm{A}_{2} .
\end{aligned}
$$

With an argument similar to that used in the estimate for $\mathrm{I}_{1}$ in [7, Theorem 3.9], it is not difficult to get

$$
\mathrm{A}_{1} \leq C\|b\|_{\widetilde{\operatorname{RBMO}}(\mu)} M_{r, 5}^{(\alpha)} f(x) .
$$

Hence, we only need to estimate $A_{2}$. For any $y_{1}, y_{2} \in B$, by applying (1.8), (1.9), (1.16), the Hölder inequality and (2.3), we have

$$
\begin{aligned}
& \mathrm{A}_{2} \leq C \int_{\mathcal{X} \backslash 6 B} \theta\left(\frac{d\left(y_{1}, y_{2}\right)}{d\left(y_{1}, z\right)}\right) \frac{\left|b(z)-b_{B}\right||f(z)|}{\left[\lambda\left(y_{1}, d\left(y_{1}, z\right)\right)\right]^{1-\alpha}} \mathrm{d} \mu(z) \\
& \leq C \sum_{k=1}^{\infty} \int_{6^{k+1} B \backslash 6^{k} B} \theta\left(\frac{r_{B}}{d\left(c_{B}, z\right)}\right) \frac{\left|b(z)-b_{B}\right||f(z)|}{\left[\lambda\left(c_{B}, d\left(c_{B}, z\right)\right)\right]^{1-\alpha}} \mathrm{d} \mu(z) \\
& \leq C \sum_{k=1}^{\infty} \theta\left(\frac{r_{B}}{6^{k} r_{B}}\right) \frac{1}{\left[\lambda\left(c_{B}, 6^{k+1} r_{B}\right)\right]^{1-\alpha}} \int_{6^{k+1} B}\left|b(z)-b_{B}\right||f(z)| \mathrm{d} \mu(z) \\
& \leq C \sum_{k=1}^{\infty} \theta\left(\frac{r_{B}}{6^{k} r_{B}}\right) \frac{1}{\left[\lambda\left(c_{B}, 6^{k+1} r_{B}\right)\right]^{1-\alpha}}\left(\int_{6^{k+1} B}\left|b(z)-b_{6^{k+1} B}\right||f(z)| \mathrm{d} \mu(z)\right. \\
& \left.+\left|b_{6^{k+1} B}-b_{B}\right| \int_{6^{k+1} B}|f(z)| \mathrm{d} \mu(z)\right) \\
& \leq C \sum_{k=1}^{\infty} \theta\left(\frac{r_{B}}{6^{k} r_{B}}\right) \frac{1}{\left[\lambda\left(c_{B}, 6^{k+1} r_{B}\right)\right]^{1-\alpha}}\left[\left(\int_{6^{k+1} B}|f(z)|^{r} \mathrm{~d} \mu(z)\right)^{\frac{1}{r}}\right. \\
& \times\left(\int_{6^{k+1} B}\left|b(z)-b_{6^{k+1} B}\right|^{r^{\prime}} \mathrm{d} \mu(z)\right)^{\frac{1}{r^{\prime}}}+k\|b\|_{\widehat{\operatorname{RBMO}}(\mu)}\left[\mu\left(6^{k+1} B\right)\right]^{1-\frac{1}{r}} \\
& \left.\times\left(\int_{6^{k+1} B}|f(z)|^{r} \mathrm{~d} \mu(z)\right)^{\frac{1}{r}}\right] \\
& \leq C\|b\|_{\widehat{\operatorname{BBMO}}(\mu)} M_{r, 5}^{(\alpha)} f(x) \sum_{k=1}^{\infty} \theta\left(\frac{r_{B}}{6^{k} r_{B}}\right) \frac{(k+1)}{\left[\lambda\left(c_{B}, 6^{k+1} r_{B}\right)\right]^{1-\alpha}}\left[\mu\left(5 \times 6^{k+1} B\right)\right]^{1-\alpha} \\
& \leq C\|b\|_{\widetilde{\operatorname{RBMO}}(\mu)} M_{r, 5}^{(\alpha)} f(x) \sum_{k=1}^{\infty} k \theta\left(\frac{1}{6^{k}}\right) \frac{\left[\mu\left(5 \times 6^{k+1} B\right)\right]^{1-\alpha}}{\left[C\left(6^{k+1}\right)\right]^{1-\alpha}\left[\lambda\left(c_{B}, r_{B}\right)\right]^{1-\alpha}} \\
& \leq C\|b\|_{\widetilde{\operatorname{RBMO}}(\mu)} M_{r, 5}^{(\alpha)} f(x) \sum_{k=1}^{\infty} \theta\left(\frac{1}{6^{k}}\right) \frac{k C_{\lambda}^{(1-\alpha) k}}{\left[C\left(6^{k+1}\right)\right]^{1-\alpha}} \\
& \leq C\|b\|_{\widetilde{\operatorname{RBO}}(\mu)} M_{r, 5}^{(\alpha)} f(x),
\end{aligned}
$$

where we have used the fact that

$$
\left|m_{B}(b)-m_{6^{k+1} B}(b)\right| \leq C k\|b\|_{\widehat{\operatorname{RMO}}(\mu)} .
$$


Further, combining the estimates for $A_{1}$ and $A_{2}$, we conclude that

$$
\frac{1}{\mu(6 B)} \int_{B}\left|\left[b, T_{\alpha}\right] f(y)-h_{B}\right| \mathrm{d} \mu(y) \leq C\|b\|_{\widetilde{\operatorname{RMO}}(\mu)} M_{r, 5}^{(\alpha)} f(x) .
$$

This, together with (3.6), (3.7) and (3.8) in [7, Theorem 3.9], implies (3.2).

Now we turn to showing (3.3). For any two balls $B \subset S$ with $B \ni x$, let

$$
N=N_{B, S}^{(6)}+\left\lfloor\log _{\rho} 2\right\rfloor+2 .
$$

Write

$$
\begin{aligned}
\left|h_{B}-h_{S}\right|= & \left|m_{B}\left(T_{\alpha}\left(\left[b-m_{B}(b)\right] f \chi_{\mathcal{X} \backslash \frac{6}{5} B}\right)\right)-m_{S}\left(T_{\alpha}\left(\left[b-m_{S}(b)\right] f \chi_{\mathcal{X} \backslash \frac{6}{5} S}\right)\right)\right| \\
\leq & \left|m_{B}\left(T_{\alpha}\left(\left[b-m_{B}(b)\right] f \chi_{6 B \backslash \frac{6}{5} B}\right)\right)\right|+\left|m_{B}\left(T_{\alpha}\left(\left[m_{B}(b)-m_{S}(b)\right] f \chi \mathcal{X} \backslash{ }_{6 B}\right)\right)\right| \\
& +\left|m_{B}\left(T_{\alpha}\left(\left[b-m_{S}(b)\right] f \chi_{6^{N} B \backslash 6 B}\right)\right)\right|+\left|m_{S}\left(T_{\alpha}\left(\left[b-m_{S}(b)\right] f \chi_{6^{N} B \backslash \frac{6}{5} S}\right)\right)\right| \\
& +\left|m_{B}\left(T_{\alpha}\left(\left[b-m_{S}(b)\right] f \chi_{\mathcal{X} \backslash 6^{N} B}\right)\right)-m_{S}\left(T_{\alpha}\left(\left[b-m_{S}(b)\right] f \chi_{\mathcal{X} \backslash 6^{N} B}\right)\right)\right| \\
= & : \mathrm{D}_{1}+\mathrm{D}_{2}+\mathrm{D}_{3}+\mathrm{D}_{4}+\mathrm{D}_{5} .
\end{aligned}
$$

Following the proof of [3, Theorem 1] and [1, Theorem 7.6], it is not difficult to get

$$
\begin{aligned}
& \mathrm{D}_{1}+\mathrm{D}_{4} \leq C\|b\|_{\widetilde{\operatorname{RMO}}(\mu)} M_{r, 5}^{(\alpha)} f(x), \\
& \mathrm{D}_{5} \leq C\left[\widetilde{K}_{B, S}^{(6)}\right]^{2}\|b\|_{\widetilde{\mathrm{RBMO}}(\mu)} M_{r, 5}^{(\alpha)} f(x),
\end{aligned}
$$

and

$$
\mathrm{D}_{2} \leq C \widetilde{K}_{B, S}^{(6)}\|b\|_{\widetilde{\operatorname{RBMO}}(\mu)}\left[T_{\alpha}(|f|)(x)+M_{r, 5}^{(\alpha)} f(x)\right]
$$

Finally, we turn to the estimate of $\mathrm{D}_{3}$. For any $z \in B$, by applying (1.9), Definition 1.7, the Hölder inequality, (2.3) and (2.5), we can conclude that

$$
\begin{aligned}
& \left|T_{\alpha}\left(\left[b-m_{S}(b)\right] f \chi_{6^{N} B \backslash 6 B}\right)(z)\right| \\
& \leq C \int_{6^{N} B \backslash 6 B} \frac{|f(y)|}{[\lambda(z, d(z, y))]^{1-\alpha}}\left|b(y)-m_{S}(b)\right| \mathrm{d} \mu(y) \\
& \leq C \sum_{k=1}^{N-1} \int_{6^{k+1} B \backslash\left(6^{k} B\right)} \frac{|f(y)|}{[\lambda(z, d(z, y))]^{1-\alpha}}\left|b(y)-m_{S}(b)\right| \mathrm{d} \mu(y) \\
& \leq C \sum_{k=1}^{N-1} \frac{1}{\left[\lambda\left(c_{B}, 6^{k} r_{B}\right)\right]^{1-\alpha}} \int_{6^{k+1} B}|f(y)|\left|b(y)-m_{S}(b)\right| \mathrm{d} \mu(y) \\
& \leq C \sum_{k=1}^{N-1} \frac{1}{\left[\lambda\left(c_{B}, 6^{k} r_{B}\right)\right]^{1-\alpha}}\left\{\int_{6^{k+1} B}|f(y)|\left|b(y)-m_{6^{k+1} B}(b)\right| \mathrm{d} \mu(y)\right. \\
& \left.\quad+\left|m_{6^{k+1} B}(b)-m_{S}(b)\right| \int_{6^{k+1} B}|f(y)| \mathrm{d} \mu(y)\right\}
\end{aligned}
$$




$$
\begin{aligned}
& \leq C \sum_{k=1}^{N-1} \frac{1}{\left[\lambda\left(c_{B}, 6^{k} r_{B}\right)\right]^{1-\alpha}}\left\{\left(\int_{6^{k+1} B}|f(y)|^{r} \mathrm{~d} \mu(y)\right)^{\frac{1}{r}}\right. \\
& \times\left(\int_{6^{k+1} B}\left|b(y)-m_{6^{k+1} B}(b)\right|^{r^{\prime}} \mathrm{d} \mu(y)\right)^{\frac{1}{r^{\prime}}} \\
& \left.+\left|m_{6^{k+1} B}(b)-m_{S}(b)\right|\left[\mu\left(6^{k+1} B\right)\right]^{1-\frac{1}{r}}\left(\int_{6^{k+1} B}|f(y)|^{r} \mathrm{~d} \mu(y)\right)^{\frac{1}{r}}\right\} \\
& \leq C \sum_{k=1}^{N-1} \frac{1}{\left[\lambda\left(c_{B}, 6^{k} r_{B}\right)\right]^{1-\alpha}}\left\{\left(\frac{1}{\left[\mu\left(5 \times 6^{k+1} B\right)\right]^{1-\alpha r}} \int_{6^{k+1} B}|f(y)|^{r} \mathrm{~d} \mu(y)\right)^{\frac{1}{r}}\right. \\
& \times\left(\frac{1}{\mu\left(5 \times 6^{k+1} B\right)} \int_{6^{k+1} B}\left|b(y)-m_{6^{k+1} B}(b)\right|^{r^{\prime}} \mathrm{d} \mu(y)\right)^{\frac{1}{r^{\prime}}}\left[\mu\left(5 \times 6^{k+1} B\right)\right]^{1-\alpha} \\
& +\left|m_{6^{k+1} B}(b)-m_{S}(b)\right|\left(\frac{1}{\left[\mu\left(5 \times 6^{k+1} B\right)\right]^{1-\alpha r}} \int_{6^{k+1} B}|f(y)|^{r} \mathrm{~d} \mu(y)\right)^{\frac{1}{r}} \\
& \left.\times\left[\mu\left(5 \times 6^{k+1}\right)\right]^{\frac{1}{r}-\alpha}\left[\mu\left(6^{k+1} B\right)\right]^{1-\frac{1}{r}}\right\} \\
& \leq C\|b\|_{\widetilde{\operatorname{RBMO}}(\mu)} M_{r, 5}^{(\alpha)}(f)(x) \sum_{k=1}^{N-1}\left[\frac{6^{k+1} B}{\lambda\left(c_{B}, 6^{k} r_{B}\right)}\right]^{1-\alpha} \widetilde{K}_{B, S}^{(\rho)} \\
& \leq C\|b\|_{\widetilde{\operatorname{RBMO}}(\mu)} M_{r, 5}^{(\alpha)}(f)(x) \widetilde{K}_{B, S}^{(\rho)} \widetilde{K}_{B, S}^{\alpha} .
\end{aligned}
$$

Further, by applying the definition of $m_{B}(f)$, we get

$$
\begin{aligned}
\mathrm{D}_{3} & =\left|\frac{1}{\mu(B)} \int_{B} T_{\alpha}\left(\left[b-m_{S}(b)\right] f \chi_{6^{N} B \backslash 6 B}\right)(z) \mathrm{d} \mu(z)\right| \\
& \leq \frac{1}{\mu(B)} \int_{B}\left|T_{\alpha}\left(\left[b-m_{S}(b)\right] f \chi_{6^{N} B \backslash 6 B}\right)(z)\right| \mathrm{d} \mu(z) \\
& \leq C\|b\|_{\widehat{\operatorname{BMMO}}(\mu)} M_{r, 5}^{(\alpha)}(f)(x) \widetilde{K}_{B, S}^{(\rho)} \widetilde{K}_{B, S}^{\alpha} .
\end{aligned}
$$

Thus, combining the estimates $\mathrm{D}_{1}, \mathrm{D}_{4}$ and $\mathrm{D}_{5}$, we complete the estimate for (3.3).

If $B$ is a doubling ball and $x \in B$, by (3.2), we have

$$
\left|m_{B}\left(\left[b, T_{\alpha}\right] f\right)-h_{B}\right| \leq C\|b\|_{\overparen{\operatorname{RMO}}(\mu)}\left[M_{r, 5}^{(\alpha)} f(x)+M_{r, 6}\left(T_{\alpha} f\right)(x)\right] .
$$

For any ball $B$ with $x \in B, \widetilde{K}_{B, \widetilde{B}}^{(\rho)} \leq C$ and $\widetilde{K}_{B, \widetilde{B}}^{\alpha} \leq C$, by (3.2), (3.3) and (3.4), we can get

$$
\begin{aligned}
& \frac{1}{\mu(6 B)} \int_{B}\left|\left[b, T_{\alpha}\right] f(y)-m_{\widetilde{B}}\left(\left[b, T_{\alpha}\right] f\right)\right| \mathrm{d} \mu(y) \\
& \quad \leq \frac{1}{\mu(6 B)} \int_{B}\left|\left[b, T_{\alpha}\right] f(y)-h_{B}\right| \mathrm{d} \mu(y)+\left|h_{B}-h_{\widetilde{B}}\right|+\left|h_{\widetilde{B}}-m_{\widetilde{B}}\left(\left[b, T_{\alpha}\right] f\right)\right| \\
& \quad \leq C\|b\|_{\widetilde{\operatorname{BMO}}(\mu)}\left\{M_{r, 5}^{(\alpha)} f(x)+M_{r, 6}\left(T_{\alpha} f\right)(x)+T_{\alpha}(|f|)(x)\right\} .
\end{aligned}
$$

Similar to (3.3), for all doubling balls $B \subset S$ with $x \in B$ such that $\widetilde{K}_{B, S}^{\alpha} \leq \widetilde{P}_{\alpha}$, we have

$$
\left|h_{B}-h_{S}\right| \leq C \widetilde{K}_{B, S}^{(\rho)}\|b\|_{\overparen{\operatorname{RBO}}(\mu)}\left[M_{r, 5}^{(\alpha)} f(x)+T_{\alpha}(|f|)(x)\right] \widetilde{P}_{\alpha} .
$$


Thus, by applying Lemma 2.8 , we know that, for all doubling $B \subset S$ with $x \in B$,

$$
\left|h_{B}-h_{S}\right| \leq C \widetilde{K}_{B, S}^{\alpha}\|b\|_{\widetilde{\operatorname{RBO}}(\mu)}\left[M_{r, 5}^{(\alpha)} f(x)+T_{\alpha}(|f|)(x)\right]
$$

and, using (3.4), we can get

$$
\begin{aligned}
& \left|m_{B}\left(\left[b, T_{\alpha}\right] f\right)-m_{S}\left(\left[b, T_{\alpha}\right] f\right)\right| \\
& \quad \leq\left|m_{B}\left(\left[b, T_{\alpha}\right] f\right)-h_{B}\right|+\left|h_{B}-h_{S}\right|+\left|h_{S}-m_{S}\left(\left[b, T_{\alpha}\right] f\right)\right| \\
& \quad \leq C \widetilde{K}_{B, S}^{\alpha}\|b\|_{\overparen{\operatorname{BMO}}(\mu)}\left\{M_{r, 5}^{(\alpha)} f(x)+M_{r, 6}\left(T_{\alpha} f\right)(x)+T_{\alpha}(|f|)(x)\right\} .
\end{aligned}
$$

Combining (3.5) and the above inequality, we can get (3.1). Hence, the proof of Theorem 3.1 is completed.

\section{Boundedness of $\left[b, T_{\alpha}\right]$ on Hardy space with discrete coefficient}

As is well known, the dual space of atomic Hardy space $\widetilde{H}_{\mathrm{atb}, \rho}^{1, q, \gamma}(\mu)$, which was introduced by Fu et al. [6], is the space $\widetilde{\mathrm{RBMO}}(\mu)$ associated with the discrete coefficient $\widetilde{K}_{B, S}^{(\rho)}$. But,in this section, we will consider the boundedness of the commutator $\left[b, T_{\alpha}\right]$ on the atomic Hardy space $\widetilde{H}_{\mathrm{atb}, b, \rho}^{1, q, \gamma}(\mu)$ which is the subspace of the $\widetilde{H}_{\mathrm{atb}, \rho}^{1, q, \gamma}(\mu)$. First, we give the definition of the atomic Hardy space $\widetilde{H}_{\text {atb,b, }, \rho}^{1, q, \gamma}(\mu)$ being slightly modified in [15] and [6].

Definition 4.1 Let $\rho \in(1, \infty), q \in(1, \infty]$ and $\gamma \in[1, \infty)$. Suppose that $b \in \widetilde{\operatorname{RBMO}}(\mu)$. A function $h \in L^{1}(\mu)$ is called a $(b, q, \gamma, \rho)_{\lambda}$-atomic block if

(1) there exists a ball $B$ such that $\operatorname{supp}(h) \subset B$;

(2) $\int_{\mathcal{X}} h(x) \mathrm{d} \mu(x)=0$, and $\int_{\mathcal{X}} b(x) h(x) \mathrm{d} \mu(x)=0$;

(3) for any $i \in\{1,2\}$, there exist a function $a_{i}$ supported on a ball $B_{i} \subset B$ and a number $\kappa_{i} \in \mathbb{C}$ such that $h=\kappa_{1} a_{1}+\kappa_{2} a_{2}$ and

$$
\left\|a_{i}\right\|_{L^{q}(\mu)} \leq\left[\mu\left(\rho B_{i}\right)\right]^{\frac{1}{q}-1}\left[\widetilde{K}_{B_{i}, B}^{(\rho)}\right]^{-\gamma} .
$$

Moreover, let

$$
|h|_{\widetilde{H}_{\mathrm{atb}, b, \rho}^{1, q, \gamma}(\mu)}:=\left|\kappa_{1}\right|+\left|\kappa_{2}\right|
$$

A function $f \in L^{1}(\mu)$ is said to belong to the atomic Hardy space $\widetilde{H}_{\mathrm{atb}, b, \rho}^{1, q, \gamma}(\mu)$ if there exist $(b, q, \gamma, \rho)_{\lambda}$-atomic blocks $\left\{h_{i}\right\}_{i=1}^{\infty}$ such that $f=\sum_{i=1}^{\infty} h_{i}$ in $L^{1}(\mu)$ and

$$
\sum_{i=1}^{\infty}\left|h_{i}\right|_{\widetilde{H}_{\mathrm{atb}, b, \rho}^{1, q, \gamma}(\mu)}<\infty
$$

The $\widetilde{H}_{\mathrm{atb}, b, \rho}^{1, q, \gamma}(\mu)$ norm of $f$ is defined by

$$
\|f\|_{\widetilde{H}_{\mathrm{atb}, b, \rho}^{1, q, \gamma}(\mu)}:=\inf \left\{\sum_{i=1}^{\infty}\left|h_{i}\right|_{\widetilde{H}_{\mathrm{atb}, b, \rho}^{1, q, \gamma}(\mu)}\right\}
$$

where the infimum is taken over all the possible decompositions of $f$ as above. 
With an argument similar to that used in [6], it is not difficult to show that, for any $q \in(1, \infty]$, the atomic Hardy space $\widetilde{H}_{\text {atb } b, \rho, \rho}^{1, q, \gamma}(\mu)$ is independent of the choices of $\rho$ and $\gamma$ and that, for all $q \in(1, \infty)$, the spaces $\widetilde{H}_{\text {atb, }, b, \rho}^{1, \rho}(\mu)$ and $\widetilde{H}_{\text {atb } b, \rho, \rho}^{1, \infty, \gamma}(\mu)$ coincide with equivalent norms. Thus, in this section, we denote the space $\widetilde{H}_{\text {atb,b, }}^{1, q, \gamma}(\mu)$ by $\widetilde{H}_{b}^{1}(\mu)$.

The main theorems of this section are stated as follows.

Theorem 4.2 Let $b \in \widetilde{\mathrm{RBMO}}(\mu), \alpha \in(0,1)$ and $T_{\alpha}$ be as in (1.11) with kernel $K_{\alpha}$ satisfying (1.9) and (1.10). Suppose that $T_{\alpha}$ is bounded on $L^{2}(\mu)$. Then there exists a positive constant C such that, for all $f \in \widetilde{H}_{b}^{1}(\mu)$,

$$
\left\|\left[b, T_{\alpha}\right] f\right\|_{L^{1-\alpha}(\mu)} \leq C\|b\|_{\widetilde{\operatorname{RBM}}(\mu)}\|f\|_{\widetilde{H}_{b}^{1}(\mu)} .
$$

Now we give the proof of Theorem 4.2.

Proof of Theorem 4.2. Without loss of generality, we may assume that $\rho=6$ as in (4.1) and $q_{0}:=\frac{1}{1-\alpha}$ for $\alpha \in(0,1)$. For any function $f \in \widetilde{H}_{b}^{1}(\mu)$, by Definition 4.1 , we can get a decomposition

$$
f(x)=\sum_{i=1}^{\infty} h_{i}(x)
$$

where $h_{i}$ is an $(\infty, 2,2)$-atomic block, $\operatorname{supp}\left(h_{i}\right) \subset S_{i}$ and

$$
\sum_{i=1}^{\infty}\left|h_{i}\right|_{\widetilde{H}_{\mathrm{atb}, 6}^{1, \infty, 2}(\mu)} \leq 2\|f\|_{\widetilde{H}_{b}^{1}(\mu)} \cdot
$$

Moreover, for each fixed $i$, we can further decompose the atomic block $h_{i}$ as

$$
h_{i}:=\kappa_{i, 1} a_{i, 1}+\kappa_{i, 2} a_{i, 2},
$$

where, for any $j \in\{1,2\}, \kappa_{i, j} \in \mathbb{C}, a_{i, j}$ is a bounded function supported on some ball $B_{i, j} \subset S_{i}$ satisfying

$$
\left\|a_{i, j}\right\|_{L^{\infty}(\mu)} \leq\left\{\mu\left(2 B_{i, j}\right)\left[\widetilde{K}_{B_{i, j}}^{(2)} S_{i}\right]^{2}\right\}^{-1},
$$

and $\left|h_{i}\right|_{\widetilde{H}_{\mathrm{atb}, b, 2}^{1, \infty, 2}(\mu)}=\left|\kappa_{i, 1}\right|+\left|\kappa_{i, 2}\right|$. Write

$$
\begin{aligned}
& \int_{\mathcal{X}}\left|\left[b, T_{\alpha}\right] f(x)\right|^{q} \mathrm{~d} \mu(x) \\
& \quad \leq C \sum_{i=1}^{\infty} \int_{\mathcal{X}}\left|\left[b, T_{\alpha}\right] h_{i}(x)\right|^{q} \mathrm{~d} \mu(x) \\
& \leq C \sum_{i=1}^{\infty} \int_{2 S_{i}}\left|\left[b, T_{\alpha}\right] h_{i}(x)\right|^{q} \mathrm{~d} \mu(x)+C \sum_{i=1}^{\infty} \int_{\mathcal{X} \backslash 2 S_{i}}\left|\left[b, T_{\alpha}\right] h_{i}(x)\right|^{q} \mathrm{~d} \mu(x) \\
& =: C \sum_{i=1}^{\infty}\left(\mathrm{F}_{i}^{1}+\mathrm{F}_{i}^{2}\right) .
\end{aligned}
$$


For $\mathrm{F}_{i}^{1}$, by applying (4.2), write

$$
\begin{aligned}
\mathrm{F}_{i}^{1} \leq & \sum_{j=1}^{2}\left|\kappa_{i, j}\right|^{q} \int_{2 S_{i}}\left|\left[b, T_{\alpha}\right] a_{i, j}(x)\right|^{q} \mathrm{~d} \mu(x) \\
\leq & C \sum_{j=1}^{2}\left|\kappa_{i, j}\right|^{q} \int_{2 B_{i, j}}\left|\left[b, T_{\alpha}\right] a_{i, j}(x)\right|^{q} \mathrm{~d} \mu(x) \\
& +C \sum_{j=1}^{2}\left|\kappa_{i, j}\right|^{q} \int_{2 S_{i} \backslash\left(2 B_{i, j}\right)}\left|\left[b, T_{\alpha}\right] a_{i, j}(x)\right|^{q} \mathrm{~d} \mu(x) \\
= & : \mathrm{F}_{i}^{1,1}+\mathrm{F}_{i}^{1,2} .
\end{aligned}
$$

Choosing suitable $p_{1}, q_{1}$, such that $1<p_{1}<\frac{1}{\alpha}, 1<q<q_{1}$ and $\frac{1}{q_{1}}=\frac{1}{p_{1}}-\alpha$. Applying the Hölder inequality, $\widetilde{K}_{B_{i, j}, S_{i}}^{(2)} \geq 1$ and Theorem 3.1 , we can deduce that

$$
\begin{aligned}
\mathrm{F}_{i}^{1,1} & \leq C \sum_{j=1}^{2}\left|\kappa_{i, j}\right|^{q}\left(\int_{2 B_{i, j}}\left|\left[b, T_{\alpha}\right] a_{i, j}(x)\right|^{q \times \frac{q_{1}}{q}} \mathrm{~d} \mu(x)\right)^{\frac{q}{q_{1}}}\left[\mu\left(2 B_{i, j}\right)\right]^{1-\frac{q}{q_{1}}} \\
& \leq C \sum_{j=1}^{2}\left|\kappa_{i, j}\right|^{q}\left(\int_{2 B_{i, j}}\left|\left[b, T_{\alpha}\right] a_{i, j}(x)\right|^{q_{1}} \mathrm{~d} \mu(x)\right)^{\frac{q}{q_{1}}}\left[\mu\left(2 B_{i, j}\right)\right]^{1-\frac{q}{q_{1}}} \\
& \leq C\|b\|_{\widetilde{\operatorname{RBMO}}(\mu)}^{q}\left(\sum_{j=1}^{2}\left|\kappa_{i, j}\right|^{q}\left\|a_{i, j}\right\|_{L^{p_{1}(\mu)}}^{q}\left[\mu\left(2 B_{i, j}\right)\right]^{1-\frac{q}{q_{1}}}\right) \\
& \leq C\|b\|_{\widetilde{\operatorname{RBMO}}(\mu)}^{q}\left(\sum_{j=1}^{2}\left|\kappa_{i, j}\right|^{q}\left\|a_{i, j}\right\|_{L^{\infty}(\mu)}^{q}\left[\mu\left(2 B_{i, j}\right)\right]^{\frac{q}{p_{1}}}\left[\mu\left(2 B_{i, j}\right)\right]^{1-\frac{q}{q_{1}}}\right) \\
& \leq C\|b\|_{\widetilde{\operatorname{RBMO}}(\mu)}^{q}\left(\sum_{j=1}^{2}\left|\kappa_{i, j}\right|^{q}\left\|a_{i, j}\right\|_{L^{\infty}(\mu)}^{q}\left[\mu\left(2 B_{i, j}\right)\right]^{1+\frac{q}{p_{1}}-\frac{q}{q_{1}}}\right) \\
& \leq C\|b\|_{\widetilde{\operatorname{RBMO}}(\mu)}^{q}\left(\sum_{j=1}^{2}\left|\kappa_{i, j}\right|^{q}\left\|a_{i, j}\right\|_{L^{\infty}(\mu)}^{q}\left[\mu\left(2 B_{i, j}\right)\right]^{1+\alpha q}\right) \\
& \leq C\|b\|_{\widetilde{\operatorname{RBMO}}(\mu)}^{q}\left(\sum_{j=1}^{2}\left|\kappa_{i, j}\right|^{q}\right) .
\end{aligned}
$$

Denote $N_{1}:=N_{2 B_{i, j}, 2 S_{i}}$. For $\mathrm{F}_{i}^{1,2}$, by (1.1), (1.9), the Minkowski inequality, (2.3) and the fact that $\left\|a_{i, j}\right\|_{L^{\infty}(\mu)} \leq\left[\mu\left(2 B_{i, j}\right)\right]^{-1}\left[\widetilde{K}_{B_{i, j}, S_{i}}^{(2)}\right]^{-2}$, we can deduce that

$$
\begin{aligned}
\mathrm{F}_{i}^{1,2} & \leq C \sum_{j=1}^{2}\left|\kappa_{i, j}\right| \int_{2 S_{i} \backslash\left(2 B_{i, j}\right)}\left|\int_{B_{i, j}} K_{\alpha}(x, y)(b(x)-b(y)) a_{i, j}(y) \mathrm{d} \mu(y)\right|^{q} \mathrm{~d} \mu(x) \\
& \leq C \sum_{j=1}^{2}\left|\kappa_{i, j}\right|^{q} \int_{2 S_{i} \backslash\left(2 B_{i, j}\right)}\left(\int_{B_{i, j}} \frac{|b(x)-b(y)|\left|a_{i, j}(y)\right|}{\lambda(x, d(x, y))} \mathrm{d} \mu(y)\right)^{q} \mathrm{~d} \mu(x) \\
& \leq C \sum_{j=1}^{2}\left|\kappa_{i, j}\right|^{q} \int_{2 S_{i} \backslash\left(2 B_{i, j}\right)}\left(\int_{B_{i, j}} \frac{\left|b(x)-m_{B_{i, j}}(b)\right|\left|a_{i, j}(y)\right|}{[\lambda(x, d(x, y))]^{1-\alpha}} \mathrm{d} \mu(y)\right)^{q} \mathrm{~d} \mu(x)
\end{aligned}
$$


Lu Journal of Inequalities and Applications

(2020) 2020:202

Page 16 of 23

$$
\begin{aligned}
& +C \sum_{j=1}^{2}\left|\kappa_{i, j}\right|^{q} \int_{2 S_{i} \backslash\left(2 B_{i, j}\right)}\left(\int_{B_{i, j}} \frac{\left|b(y)-m_{B_{i, j}}(b)\right|\left|a_{i, j}(y)\right|}{[\lambda(x, d(x, y))]^{1-\alpha}} \mathrm{d} \mu(y)\right)^{q} \mathrm{~d} \mu(x) \\
& \leq C \sum_{j=1}^{2}\left|\kappa_{i, j}\right|^{q}\left\{\int_{B_{i, j}}\left|a_{i, j}(y)\right|\left(\int_{2 S_{i} \backslash\left(2 B_{i, j}\right)} \frac{\left|b(x)-m_{B_{i, j}}(b)\right|^{q}}{\lambda(x, d(x, y))} \mathrm{d} \mu(x)\right)^{\frac{1}{q}} \mathrm{~d} \mu(y)\right\}^{q} \\
& +C \sum_{j=1}^{2}\left|\kappa_{i, j}\right|^{q}\left\{\int_{B_{i, j}}\left|b(y)-m_{B_{i, j}}(b)\right|\left|a_{i, j}(y)\right|\left(\int_{2 S_{i} \backslash\left(2 B_{i, j}\right)} \frac{\mathrm{d} \mu(x)}{\lambda(x, d(x, y))}\right)^{\frac{1}{q}} \mathrm{~d} \mu(y)\right\}^{q} \\
& \leq C \sum_{j=1}^{2}\left|\kappa_{i, j}\right|^{q}\left\{\int_{B_{i, j}}\left|a_{i, j}(y)\right|\left(\sum_{k=1}^{N_{1}-1} \int_{2^{k+1} B_{i, j} \backslash\left(2^{k} B_{i, j}\right)} \frac{\left|b(x)-m_{B_{i, j}}(b)\right|^{q}}{\lambda\left(c_{B_{i, j}}, d\left(x, c_{B_{i, j}}\right)\right)} \mathrm{d} \mu(x)\right)^{\frac{1}{q}} \mathrm{~d} \mu(y)\right\}^{q} \\
& +C \sum_{j=1}^{2}\left|\kappa_{i, j}\right|^{q}\left\{\int_{B_{i, j}}\left|b(y)-m_{B_{i, j}}(b)\right|\left|a_{i, j}(y)\right|\right. \\
& \left.\times\left(\int_{2 S_{i} \backslash\left(2 B_{i, j}\right)} \frac{\mathrm{d} \mu(x)}{\lambda\left(c_{B_{i, j}}, d\left(c_{B_{i, j}}, x\right)\right)}\right)^{\frac{1}{q}} \mathrm{~d} \mu(y)\right\}^{q} \\
& \leq C \sum_{j=1}^{2}\left|\kappa_{i, j}\right|^{q}\left\{\left(\sum_{k=1}^{N_{1}-1} \frac{1}{\lambda\left(c_{B_{i, j}}, 2^{k} r_{B_{i, j}}\right)} \int_{2^{k+1} B_{i, j}}\left|b(x)-m_{B_{i, j}}(b)\right|^{q} \mathrm{~d} \mu(x)\right)^{\frac{1}{q}}\right. \\
& \left.\times \int_{B_{i, j}}\left|a_{i, j}(y)\right| \mathrm{d} \mu(y)\right\}^{q} \\
& +C \sum_{j=1}^{2}\left|\kappa_{i, j}\right|^{q}\left\{\int_{B_{i, j}}\left|b(y)-m_{B_{i, j}}(b)\right|\left|a_{i, j}(y)\right| \mathrm{d} \mu(y)\right\}^{q}\left(\sum_{k=1}^{N_{1}-1} \frac{\mu\left(2^{k+1} B_{i, j}\right)}{\lambda\left(c_{B_{i, j}}, 2^{k+1} r_{B_{i, j}}\right)}\right) \\
& \leq C\|b\|_{\overparen{\operatorname{RBMO}}(\mu)}^{q} \sum_{j=1}^{2}\left|\kappa_{i, j}\right|^{q}\left(\int_{B_{i, j}}\left|a_{i, j}(y)\right| \mathrm{d} \mu(y)\right)^{q} \\
& \times\left(\sum_{k=1}^{N_{1}-1} \frac{\mu\left(2^{k+1} B_{i, j}\right)}{\lambda\left(c_{B_{i, j}}, 2^{k+1} r_{B_{i, j}}\right)}\right) \\
& +C \sum_{j=1}^{2}\left|\kappa_{i, j}\right|^{q}\left\{\int_{B_{i, j}}\left|b(y)-m_{B_{i, j}}(b)\right|\left|a_{i, j}(y)\right| \mathrm{d} \mu(y)\right\}^{q}\left(\sum_{k=1}^{N_{1}-1} \frac{\mu\left(2^{k+1} B_{i, j}\right)}{\lambda\left(c_{B_{i, j}}, 2^{k+1} r_{B_{i, j}}\right)}\right) \\
& \leq C\|b\|_{\widetilde{\operatorname{RMO}}(\mu)}^{q} \sum_{j=1}^{2}\left|\kappa_{i, j}\right|^{q}\left\|a_{i, j}\right\|_{L^{\infty}(\mu)}^{q}\left[\mu\left(B_{i, j}\right)\right]^{q} \widetilde{K}_{B_{i, j}, S_{i}}^{(2)} \\
& +C \sum_{j=1}^{2}\left|\kappa_{i, j}\right|^{q}\left\|a_{i, j}\right\|_{L^{\infty}(\mu)}^{q}\left(\int_{B_{i, j}}\left|b(y)-m_{B_{i, j}}(b)\right|\left|a_{i, j}(y)\right| \mathrm{d} \mu(y)\right)^{q} \widetilde{K}_{B_{i, j}, S_{i}}^{(2)} \\
& \leq C\|b\|_{\overparen{\operatorname{RBMO}}(\mu)}^{q} \sum_{j=1}^{2}\left|\kappa_{i, j}\right|^{q}\left\|a_{i, j}\right\|_{L^{\infty}(\mu)}^{q}\left[\mu\left(B_{i, j}\right)\right]^{q} \widetilde{K}_{B_{i, j}, S_{i}}^{(2)} \\
& \leq C\|b\|_{\overparen{\operatorname{RBMO}}(\mu)}^{q} \sum_{j=1}^{2}\left|\kappa_{i, j}\right|^{q}\left[\mu\left(B_{i, j}\right)\right]^{q} \widetilde{K}_{B_{i, j}, S_{i}}^{(2)}\left[\mu\left(2 B_{i, j}\right)\right]^{-q}\left[\widetilde{K}_{B_{i, j}, S_{i}}^{(2)}\right]^{-2 q} \\
& \leq C\|b\|_{\overparen{\operatorname{RBO}(\mu)}}^{q}\left(\sum_{j=1}^{2}\left|\kappa_{i, j}\right|^{q}\right) \text {. }
\end{aligned}
$$


Combining the estimates for $\mathrm{F}_{i}^{1,1}$ and $\mathrm{F}_{i}^{1,2}$, we have

$$
\mathrm{F}_{i}^{1} \leq C\|b\|_{\overparen{\operatorname{RBMO}}(\mu)}^{q}\left(\sum_{j=1}^{2}\left|\kappa_{i, j}\right|^{q}\right) .
$$

Now we turn to $F_{i}^{2}$, by applying the vanishing condition of $h_{i}$, the Hölder inequality, (1.10), (1.16) and $y \in S_{i}$, we can get

$$
\begin{aligned}
& \mathrm{F}_{i}^{2}=\int_{\mathcal{X} \backslash 2 S_{i}}\left|\int_{\mathcal{X}} K_{\alpha}(x, y)(b(x)-b(y)) h_{i}(y) \mathrm{d} \mu(y)\right|^{q} \mathrm{~d} \mu(x) \\
& =\int_{\mathcal{X} \backslash 2 S_{i}} \mid \int_{\mathcal{X}} K_{\alpha}(x, y)(b(x)-b(y)) h_{i}(y) \mathrm{d} \mu(y) \\
& -\left.\int_{\mathcal{X}} K_{\alpha}\left(x, c_{S_{i}}\right)(b(x)-b(y)) h_{i}(y) \mathrm{d} \mu(y)\right|^{q} \mathrm{~d} \mu(x) \\
& \leq \int_{\mathcal{X} \backslash 2 S_{i}}\left(\int_{S_{i}}\left|K_{\alpha}(x, y)-K_{\alpha}\left(x, c_{S_{i}}\right)\right||b(x)-b(y)|\left|h_{i}(y)\right| \mathrm{d} \mu(y)\right)^{q} \mathrm{~d} \mu(x) \\
& \leq\left\{\int_{S_{i}}\left|h_{i}(y)\right|\left(\int_{\mathcal{X} \backslash 2 S_{i}}\left|K_{\alpha}(x, y)-K_{\alpha}\left(x, c_{S_{i}}\right)\right|^{q}|b(x)-b(y)|^{q} \mathrm{~d} \mu(x)\right)^{\frac{1}{q}} \mathrm{~d} \mu(y)\right\}^{q} \\
& \leq C\left\{\int_{S_{i}}\left|h_{i}(y)\right|\left[\int_{\mathcal{X} \backslash 2 S_{i}}\left[\theta\left(\frac{d\left(y, c_{S_{i}}\right)}{d(x, y)}\right)\right]^{q} \frac{\left|b(x)-m_{S_{i}}(b)\right|^{q}}{\lambda(y, d(x, y))} \mathrm{d} \mu(x)\right]^{\frac{1}{q}} \mathrm{~d} \mu(y)\right. \\
& \left.+\int_{S_{i}}\left|h_{i}(y)\right|\left|b(y)-m_{S_{i}}(b)\right|\left[\int_{\mathcal{X} \backslash 2 S_{i}}\left[\theta\left(\frac{d\left(y, c_{S_{i}}\right)}{d(x, y)}\right)\right]^{q} \frac{\mathrm{d} \mu(x)}{\lambda(y, d(x, y))}\right]^{\frac{1}{q}} \mathrm{~d} \mu(y)\right\}^{q} \\
& \leq C\left\{\int_{S_{i}}\left|h_{i}(y)\right|\left[\sum_{k=1}^{\infty} \int_{2^{k+1} S_{i} \backslash 2^{k} S_{i}}\left[\theta\left(\frac{r_{S_{i}}}{d\left(x, c_{S_{i}}\right)}\right)\right]^{q} \frac{\left|b(x)-m_{S_{i}}(b)\right|^{q}}{\lambda\left(c_{S_{i}}, d\left(x, c_{S_{i}}\right)\right)} \mathrm{d} \mu(x)\right]^{\frac{1}{q}} \mathrm{~d} \mu(y)\right. \\
& +\int_{S_{i}}\left|h_{i}(y)\right|\left|b(y)-m_{S_{i}}(b)\right| \\
& \left.\times\left[\sum_{k=1}^{\infty} \int_{2^{k+1} S_{i} \backslash 2^{k} S_{i}}\left[\theta\left(\frac{r_{S_{i}}}{d\left(x, c_{S_{i}}\right)}\right)\right]^{q} \frac{\mathrm{d} \mu(x)}{\lambda\left(c_{S_{i}}, d\left(x, c_{S_{i}}\right)\right)}\right]^{\frac{1}{q}} \mathrm{~d} \mu(y)\right\}^{q} \\
& \leq C\left\{\int_{S_{i}}\left|h_{i}(y)\right|\left[\sum_{k=1}^{\infty} \int_{2^{k+1} S_{i}} \frac{1}{\lambda\left(c_{S_{i}}, 2^{k} r_{S_{i}}\right)}\left[\theta\left(\frac{r_{S_{i}}}{2^{k} r_{S_{i}}}\right)\right]^{q}\left|b(x)-m_{S_{i}}(b)\right|^{q} \mathrm{~d} \mu(x)\right]^{\frac{1}{q}} \mathrm{~d} \mu(y)\right. \\
& \left.+\int_{S_{i}}\left|h_{i}(y)\right|\left|b(y)-m_{S_{i}}(b)\right|\left(\sum_{k=1}^{\infty}\left[\theta\left(\frac{r_{S_{i}}}{2^{k} r_{S_{i}}}\right)\right]^{q} \frac{\mu\left(2^{k+1} S_{i}\right)}{\lambda\left(c_{S_{i}}, 2^{k} r_{S_{i}}\right)}\right)^{\frac{1}{q}} \mathrm{~d} \mu(y)\right\}^{q} \\
& \leq C\left\{\int _ { S _ { i } } | h _ { i } ( y ) | \mathrm { d } \mu ( y ) \left(\sum_{k=1}^{\infty} \frac{\mu\left(2^{k+2} S_{i}\right)}{\lambda\left(c_{S_{i}}, 2^{k} r_{S_{i}}\right)}\left[\theta\left(\frac{1}{2^{k}}\right)\right]^{q}\right.\right. \\
& \left.\times \frac{1}{\mu\left(2^{k+2} S_{i}\right)} \int_{2^{k+1} S_{i}}\left|b(x)-m_{S_{i}}(b)\right|^{q} \mathrm{~d} \mu(x)\right)^{\frac{1}{q}} \\
& \left.+\int_{S_{i}}\left|h_{i}(y)\right|\left|b(y)-m_{S_{i}}(b)\right| \mathrm{d} \mu(y)\left[\sum_{k=1}^{\infty} \theta\left(\frac{1}{2^{k}}\right)\right]\right\}^{q}
\end{aligned}
$$




$$
\begin{aligned}
& \leq C\left\{\int_{S_{i}}\left|h_{i}(y)\right|\left|b(y)-m_{S_{i}}(b)\right| \mathrm{d} \mu(y)+\int_{S_{i}}\left|h_{i}(y)\right| \mathrm{d} \mu(y)\left(\sum_{k=1}^{\infty} \frac{\mu\left(2^{k+2} S_{i}\right)}{\lambda\left(c_{S_{i}}, 2^{k} r_{S_{i}}\right)}\left[\theta\left(\frac{1}{2^{k}}\right)\right]^{q}\right.\right. \\
& \left.\left.\times \frac{1}{\mu\left(2^{k+2} S_{i}\right)} \int_{2^{k+1} S_{i}}\left(\left|b(x)-m_{2^{k+1} S_{i}}(b)\right|+\left|m_{2^{k+1} S_{i}}(b)-m_{S_{i}}(b)\right|\right)^{q} \mathrm{~d} \mu(x)\right)^{\frac{1}{q}}\right\}^{q} \\
& \leq C\left\{\sum_{j=1}^{2}\left|\kappa_{i, j}\right| \int_{B_{i, j}}\left|a_{i, j}(y)\right|\left|b(y)-m_{S_{i}}(b)\right| \mathrm{d} \mu(y)\right. \\
& \left.+\|b\|_{\overparen{\operatorname{RBMO}}(\mu)} \int_{S_{i}}\left|h_{i}(y)\right| \mathrm{d} \mu(y)\left(\sum_{k=1}^{\infty} \frac{\mu\left(2^{k+2} S_{i}\right)}{\lambda\left(c_{S_{i}}, 2^{k} r_{S_{i}}\right)}\left[\theta\left(\frac{1}{2^{k}}\right)\right]^{q}(k+1)^{q}\right)^{\frac{1}{q}}\right\}^{q} \\
& \leq C\|b\|_{\overparen{\operatorname{RBO}(\mu)}}^{q}\left\{\sum_{j=1}^{2}\left|\kappa_{i, j}\right|\left\|a_{i, j}\right\|_{L^{\infty}(\mu)} \mu\left(B_{i, j}\right)\right. \\
& \left.+\sum_{j=1}^{2}\left|\kappa_{i, j}\right|\left\|a_{i, j}\right\|_{L^{\infty}(\mu)}\left[\mu\left(B_{i, j}\right)\right]\left(\sum_{k=1}^{\infty} \frac{\mu\left(2^{k+2} S_{i}\right)}{\lambda\left(c_{S_{i}}, 2^{k} r_{S_{i}}\right)}\left[\theta\left(\frac{1}{2^{k}}\right)\right]^{q}(k+1)^{q}\right)^{\frac{1}{q}}\right\}^{q} \\
& \leq C\|b\|_{\widehat{\operatorname{RBO}(\mu)}}^{q}\left\{\sum_{j=1}^{2}\left|\kappa_{i, j}\right|+\sum_{j=1}^{2}\left|\kappa_{i, j}\right|\left[\sum_{k=1}^{\infty}\left(\frac{\mu\left(2^{k+2} S_{i}\right)}{\lambda\left(c_{S_{i}}, 2^{k} r_{S_{i}}\right)}\right)^{\frac{1}{q}} \theta\left(\frac{1}{2^{k}}\right)(k+1)\right]\right\}^{q} \\
& \leq C\|b\|_{\overparen{\operatorname{RBMO}}(\mu)}^{q}\left\{\sum_{j=1}^{2}\left|\kappa_{i, j}\right|+\sum_{j=1}^{2}\left|\kappa_{i, j}\right|\left[\sum_{k=1}^{\infty}\left(\frac{\mu\left(2^{k+2} S_{i}\right)}{\left[C\left(2^{k+1}\right)\right] \lambda\left(c_{S_{i}}, r_{S_{i}}\right)}\right)^{\frac{1}{q}} \theta\left(\frac{1}{2^{k}}\right) k\right]\right\}^{q} \\
& \leq C\|b\|_{\widetilde{\operatorname{RBMO}}(\mu)}^{q}\left\{\sum_{j=1}^{2}\left|\kappa_{i, j}\right|+\sum_{j=1}^{2}\left|\kappa_{i, j}\right|\left[\sum_{k=1}^{\infty}\left(\frac{k C_{\lambda}^{\frac{k}{q}}}{\left[C\left(2^{k+1}\right)\right]^{\frac{1}{q}}}\right)+\sum_{k=1}^{\infty} \theta\left(\frac{1}{2^{k}}\right)\right]\right\}^{q} \\
& \leq C\|b\|_{\overparen{\operatorname{RBMO}}(\mu)}^{q}\left(\sum_{j=1}^{2}\left|\kappa_{i, j}\right|\right)^{q} \text {, }
\end{aligned}
$$

where we have used the following inequality (see [16]):

$$
\int_{0}^{1} \frac{\theta(t)}{t} \mathrm{~d} t \geq \sum_{k=1}^{\infty} \int_{2^{-k}}^{2^{1-k}} \frac{\theta\left(2^{-k}\right)}{2^{1-k}} \mathrm{~d} t \geq C \sum_{k=1}^{\infty} \theta\left(2^{-k}\right)
$$

Combining the above estimates for $\mathrm{F}_{i}^{1}$ and $\mathrm{F}_{i}^{2}$, we can deduce that

$$
\left\|\left[b, T_{\alpha}\right] f\right\|_{L^{q}(\mu)}=\left(\int_{\mathcal{X}}\left|\left[b, T_{\alpha}\right] f(x)\right|^{q} \mathrm{~d} \mu(x)\right)^{\frac{1}{q}} \leq C \sum_{i=1}^{\infty}\left|h_{i}\right|_{\widetilde{H}_{\mathrm{atb}, b, 2}^{1, \infty, 2}(\mu)} \leq C\|f\|_{\widetilde{H}_{b}^{1}(\mu)} .
$$

Thus, we complete the proof of Theorem 4.2.

\section{Boundedness of $\left[b, T_{\alpha}\right]$ on Morrey space}

In this section, we will mainly establish the boundedness of the commutator $\left[b, T_{\alpha}\right]$ generated by $T_{\alpha}$ and the space $\widetilde{\mathrm{RBMO}}(\mu)$ on the Morrey space. Before giving the main result of this section, we first recall the definition of the Morrey space introduced by Cao and Zhou in [2]. 
Definition 5.1 ([2]) Let $k>1$. For any $1<q \leq p<\infty$, define

$$
M_{q}^{p}(\mu):=\left\{f \in L_{\mathrm{loc}}^{q}(\mu):\|f\|_{M_{q}^{p}(\mu)}<\infty\right\}
$$

where

$$
\|f\|_{M_{q}^{p}(\mu)}:=\sup _{B}[\mu(k B)]^{\frac{1}{p}-\frac{1}{q}}\left(\int_{B}|f(y)|^{q} \mathrm{~d} \mu(y)\right)^{\frac{1}{q}} .
$$

From [2], Cao and Zhou have showed that the norm $\|f\|_{M_{q}^{p}(\mu)}$ is independent of the choice of $k$ for $k>1$.

The main theorem of this section is stated as follows.

Theorem 5.2 Let $b \in \widetilde{\mathrm{RBMO}}(\mu), K_{\alpha}$ satisfy (1.9) and (1.10), $1<q \leq p<\infty, 1<t \leq s<\infty$, $\alpha \in(0,1)$, and $\frac{1}{s}=\frac{1}{p}-\alpha, \frac{s}{t}=\frac{p}{q}$. Suppose that $T_{\alpha}$ is as in (1.11). Then there exists a constant $C>0$, such that, for all $f \in M_{q}^{p}(\mu)$,

$$
\left\|\left[b, T_{\alpha}\right] f\right\|_{M_{t}^{s}(\mu)} \leq C\|b\|_{\widetilde{\operatorname{RMOO}(\mu)}}\|f\|_{M_{q}^{p}(\mu)}
$$

To prove the main theorem, we need to recall and establish the following lemmas.

Lemma 5.3 ([2]) If $1<q \leq p<\infty, 1<t \leq s<\infty, \alpha \in(0,1)$, and $\frac{1}{s}=\frac{1}{p}-\alpha, \frac{s}{t}=\frac{p}{q}$. Then the operator $M_{s, \zeta}^{(\alpha)}$ is bounded from $M_{q}^{p}(\mu)$ into $M_{t}^{s}(\mu)$.

Lemma 5.4 ([2]) If $\zeta>5$ and $1<r<q \leq p<\infty$, then the maximal operator $M_{r, \zeta}$ in Lemma 2.4 is bounded on $M_{q}^{p}(\mu)$.

Lemma 5.5 ([14]) Let $f \in L_{\text {loc }}^{1}(\mu)$ satisfying that $\int_{\mathcal{X}} f(x) \mathrm{d} \mu(x)=0$ when $\|\mu\|:=\mu(\mathcal{X})<\infty$. Assume that, for some $p, q$ satisfying $1<q \leq p<\infty$, $\inf \{1, N f\} \in M_{q}^{p}(\mu)$. Then there exists a constant $C>0$, independent off, such that $\|N f\|_{M_{q}^{p}(\mu)} \leq C\left\|M^{\sharp, \alpha} f\right\|_{M_{q}^{p}(\mu)}$.

Lemma 5.6 Let $K_{\alpha}$ satisfy (1.9), $1<q \leq p<\infty, 1<t \leq s<\infty, \alpha \in(0,1)$, and $\frac{1}{s}=\frac{1}{p}-\alpha$, $\frac{s}{t}=\frac{p}{q}$. Then there exists a positive constant $C$, such that, for all $f \in M_{q}^{p}(\mu)$,

$$
\left\|T_{\alpha}(f)\right\|_{M_{t}^{s}(\mu)} \leq C\|f\|_{M_{q}^{p}(\mu)} .
$$

Proof With an argument similar to that used in the proof of Lemma 3.2, it is not difficult to obtain

$$
\left|T_{\alpha} f(x)\right| \leq C I_{\alpha}(|f|)(x), \quad x \in \mathcal{X}
$$

By applying the $\left(M_{q}^{p}(\mu), M_{t}^{s}(\mu)\right)$-boundedness of $I_{\alpha}$ (see [2, Theorem 20]), we have

$$
\left\|T_{\alpha}(f)\right\|_{M_{t}^{s}(\mu)} \leq C\left\|I_{\alpha}(|f|)\right\|_{M_{t}^{s}(\mu)} \leq C\|f\|_{M_{q}^{p}(\mu)} .
$$

Hence, the proof of Lemma 5.6 is completed.

Now we give the proof of Theorem 5.2 as follows. 
Proof of Theorem 5.2 By applying Lemma 2.4, (3.1), and Lemmas 5.3-5.6, we have

$$
\begin{aligned}
\left\|\left[b, T_{\alpha}\right] f\right\|_{M_{t}^{s}(\mu)} & \leq\left\|N\left(\left[b, T_{\alpha}\right] f\right)\right\|_{M_{t}^{s}(\mu)} \leq\left\|M^{\sharp, \alpha}\left(\left[b, T_{\alpha}\right] f\right)\right\|_{M_{t}^{s}(\mu)} \\
& \leq C\|b\|_{\widetilde{\operatorname{RBM}(\mu)}}\left\{\left\|M_{r, 5}^{(\alpha)} f\right\|_{M_{t}^{s}(\mu)}+\left\|M_{r, 6}\left(T_{\alpha} f\right)\right\|_{M_{t}^{s}(\mu)}+\left\|T_{\alpha}(|f|)\right\|_{M_{t}^{s}(\mu)}\right\} \\
& \leq C\|b\|_{\widetilde{\operatorname{RBMO}}(\mu)}\left\{\|f\|_{M_{q}^{p}(\mu)}+\left\|T_{\alpha} f\right\|_{M_{t}^{s}(\mu)}+\|f\|_{M_{q}^{p}(\mu)}\right\} \\
& \leq C\|b\|_{\widetilde{\operatorname{RBMO}(\mu)}}\|f\|_{M_{q}^{p}(\mu)} .
\end{aligned}
$$

Hence, we complete the proof of Theorem 5.2.

\section{Boundedness of $\left[b, T_{\alpha}\right]$ on generalized Morrey space}

In this section, we will consider the boundedness of the commutator $\left[b, T_{\alpha}\right]$ generated by the $\widehat{\operatorname{RBMO}}(\mu)$ function and $T_{\alpha}$ on generalized Morrey space $L^{p, \phi}(\mu)$. First, we recall the following definition of the generalized Morrey space given in [14].

Definition 6.1 ([15]) Let $k>1$ and $1<p<\infty$. Suppose that $\phi:(0, \infty) \rightarrow(0, \infty)$ is an increasing function. Then the generalized Morrey space $L^{p, \phi}(\mu)$ is defined by

$$
L^{p, \phi}(\mu):=\left\{f \in L_{\mathrm{loc}}^{p}(\mu):\|f\|_{L^{p, \phi}(\mu)}<\infty\right\},
$$

where

$$
\|f\|_{L^{p, \phi}(\mu)}=\sup _{B}\left(\frac{1}{\phi(\mu(k B))} \int_{B}|f(x)|^{p} \mathrm{~d} \mu(x)\right)^{\frac{1}{p}} .
$$

From [14], Lu and Tao have showed that the norm $\|\cdot\|_{L^{p, \phi}(\mu)}$ is independent of the choice $k$ for $k>1$.

The main theorem of this section is stated as follows.

Theorem 6.2 Let $K_{\alpha}$ satisfy (1.9) and (1.10), $b \in \widehat{\operatorname{RBMO}}(\mu), 1<p<q<\infty, \frac{1}{q}=\frac{1}{p}-\alpha$ with $\alpha \in(0,1)$, and $\phi$ be an increasing function. Suppose that $T_{\alpha}$ is as in (1.11), the mapping $t \mapsto \frac{\phi(t)}{t}$ is almost decreasing and there exists a constant $C>0$ such that

$$
\frac{\phi(t)}{t} \leq C \frac{\phi(s)}{s}
$$

for $s \leq t$, and the $\lambda$ satisfies the $\left(\frac{1}{p}-\alpha\right)$-weak reverse doubling condition. Then there exists a positive constant $C$, such that, for all $f \in L^{p, \phi}(\mu)$,

$$
\left\|\left[b, T_{\alpha}\right] f\right\|_{L^{q, \phi} q / p} \leq C\|b\|_{\widetilde{\operatorname{RBMO}}(\mu)}\|f\|_{L^{p, \phi}(\mu)} .
$$

Proof Without loss of generality, we may assume that $k=8$ in (6.1). Decompose the function $f(x)$ as

$$
f(x):=f_{1}(x)+f_{2}(x),
$$


where $f_{1}(x)=f \chi_{6 B}(x)$ and $f_{2}(x)=f \chi_{\mathcal{X} \backslash 6 B}(x)$. Write

$$
\left\|\left[b, T_{\alpha}\right] f\right\|_{L^{q, \phi^{q / p}}(\mu)} \leq\left\|\left[b, T_{\alpha}\right] f_{1}\right\|_{L^{q, \phi} q_{(\mu)}}+\left\|\left[b, T_{\alpha}\right] f_{2}\right\|_{L^{q, \phi} q_{(\mu)}}=: \mathrm{E}_{1}+\mathrm{E}_{2} .
$$

By applying Lemma 3.2, we have

$$
\begin{aligned}
\mathrm{E}_{1} & =\sup _{B} \frac{1}{[\phi(\mu(8 B))]^{\frac{1}{p}}}\left(\int_{B}\left|\left[b, T_{\alpha}\right] f_{1}(x)\right|^{q} \mathrm{~d} \mu(x)\right)^{\frac{1}{q}} \\
& \leq C\|b\|_{\overparen{\operatorname{RBO}}(\mu)} \sup _{B} \frac{1}{[\phi(\mu(8 B))]^{\frac{1}{p}}}\left\|f_{1}\right\|_{L^{p}(\mu)} \\
& \leq C\|b\|_{\widetilde{\operatorname{RMO}}(\mu)}\|f\|_{L^{p}, \phi}(\mu) .
\end{aligned}
$$

For any $x \in B$, by applying (1.9), the Hölder inequality and Lemma 2.2, we can get

$$
\begin{aligned}
& \left|\left[b, T_{\alpha}\right] f_{2}(x)\right| \leq \int_{\mathcal{X} \backslash 6 B}\left|K_{\alpha}(x, y)\right||b(x)-b(y)||f(y)| \mathrm{d} \mu(y) \\
& \leq C \int_{\mathcal{X} \backslash 6 B} \frac{|f(y)|}{[\lambda(x, d(x, y))]^{1-\alpha}}|b(x)-b(y)| \mathrm{d} \mu(y) \\
& \leq C \sum_{k=1}^{\infty} \int_{6^{k+1} B \backslash 6^{k} B} \frac{|f(y)|}{[\lambda(x, d(x, y))]^{1-\alpha}}|b(x)-b(y)| \mathrm{d} \mu(y) \\
& \leq C \sum_{k=1}^{\infty} \frac{1}{\left[\lambda\left(c_{B}, 6^{k} r_{B}\right)\right]^{1-\alpha}}\left[\left|b(x)-m_{6^{k+1} B}(b)\right| \int_{6^{k+1} B}|f(y)| \mathrm{d} \mu(y)\right. \\
& \left.+\int_{6^{k+1} B}|f(y)|\left|b(y)-m_{6^{k+1} B}(b)\right| \mathrm{d} \mu(y)\right] \\
& \leq C \sum_{k=1}^{\infty} \frac{1}{\left[\lambda\left(c_{B}, 6^{k} r_{B}\right)\right]^{1-\alpha}}\left\{\left|b(x)-m_{6^{k+1} B}(b)\right|\left(\int_{6^{k+1} B}|f(y)|^{p} \mathrm{~d} \mu(y)\right)^{\frac{1}{p}}\right. \\
& \times\left[\mu\left(6^{k+1} B\right)\right]^{1-\frac{1}{p}}+\left(\int_{6^{k+1} B}|f(y)|^{p} \mathrm{~d} \mu(y)\right)^{\frac{1}{p}} \\
& \left.\times\left(\int_{6^{k+1} B}\left|b(y)-m_{6^{k+1} B}(b)\right|^{p^{\prime}} \mathrm{d} \mu(y)\right)^{\frac{1}{p^{\prime}}}\right\} \\
& \leq C\|f\|_{L^{p, \phi}(\mu)} \sum_{k=1}^{\infty} \frac{1}{\left[\lambda\left(c_{B}, 6^{k} r_{B}\right)\right]^{1-\alpha}}\left\{\left|b(x)-m_{6^{k+1} B}(b)\right|\left[\phi\left(\mu\left(2 \times 6^{k+1} B\right)\right)\right]^{\frac{1}{p}}\right. \\
& \left.\times\left[\mu\left(6^{k+1} B\right)\right]^{1-\frac{1}{p}}+\|b\|_{\overparen{\operatorname{RBMO}}(\mu)}\left[\phi\left(\mu\left(2 \times 6^{k+1} B\right)\right)\right]^{\frac{1}{p}}\left[\mu\left(6^{k+1} B\right)\right]^{1-\frac{1}{p}}\right\} \\
& \leq C\|f\|_{L^{p, \phi}(\mu)} \sum_{k=1}^{\infty} \frac{\left[\lambda\left(c_{B}, 6^{k+1} r_{B}\right)\right]^{1-\frac{1}{p}}}{\left[\lambda\left(c_{B}, 6^{k} r_{B}\right)\right]^{1-\alpha}}\left[\phi\left(\mu\left(2 \times 6^{k+1} B\right)\right)\right]^{\frac{1}{p}} \\
& \times\left\{\left|b(x)-m_{6^{k+1} B}(b)\right|+\|b\|_{\widetilde{\operatorname{RBO}}(\mu)}\right\} \\
& \leq C\|f\|_{L^{p, \phi}(\mu)} \sum_{k=1}^{\infty}\left[\lambda\left(c_{B}, 6^{k+1} r_{B}\right)\right]^{\alpha-\frac{1}{p}}\left[\phi\left(\mu\left(2 \times 6^{k+1} B\right)\right)\right]^{\frac{1}{p}} \\
& \times\left\{\left|b(x)-m_{6^{k+1} B}(b)\right|+\|b\|_{\widetilde{\operatorname{RMO}}(\mu)}\right\},
\end{aligned}
$$


furthermore, by applying Definition 6.1, (2.3) and (6.2), we can deduce that

$$
\begin{aligned}
& \mathrm{E}_{2}=\sup _{B} \frac{1}{[\phi(\mu(8 B))]^{\frac{1}{p}}}\left(\int_{B}\left|\left[b, T_{\alpha}\right]\left(f_{2}\right)(x)\right|^{q} \mathrm{~d} \mu(x)\right)^{\frac{1}{q}} \\
& \leq C\|b\|_{\overparen{\operatorname{RBO}}(\mu)}\|f\|_{L^{p, \phi}(\mu)} \sum_{k=1}^{\infty}\left[\lambda\left(c_{B}, 6^{k+1} r_{B}\right)\right]^{\alpha-\frac{1}{p}} \sup _{B} \frac{\left[\phi\left(\mu\left(2 \times 6^{k+1} B\right)\right)\right]^{\frac{1}{p}}}{[\phi(\mu(8 B))]^{\frac{1}{p}}}[\mu(B)]^{\frac{1}{p}-\alpha} \\
& +C\|f\|_{L^{p, \phi}(\mu)} \sum_{k=1}^{\infty}\left[\lambda\left(c_{B}, 6^{k+1} r_{B}\right)\right]^{\alpha-\frac{1}{p}} \sup _{B} \frac{\left[\phi\left(\mu\left(2 \times 6^{k+1} B\right)\right)\right]^{\frac{1}{p}}}{[\phi(\mu(8 B))]^{\frac{1}{p}}} \\
& \times\left(\int_{B}\left|b(x)-m_{6^{k+1} B}(b)\right|^{q} \mathrm{~d} \mu(x)\right)^{\frac{1}{q}} \\
& \leq C\|b\|_{\widetilde{\operatorname{RMO}}(\mu)}\|f\|_{L^{p, \phi}(\mu)} \sum_{k=1}^{\infty} \frac{1}{\left[C\left(6^{k+1}\right)\right]^{\frac{1}{p}-\alpha}} \sup _{B} \frac{\left[\phi\left(\mu\left(2 \times 6^{k+1} B\right)\right)\right]^{\frac{1}{p}}}{[\phi(\mu(8 B))]^{\frac{1}{p}}} \\
& +C\|f\|_{L^{p, \phi}(\mu)} \sum_{k=1}^{\infty}\left[\lambda\left(c_{B}, 6^{k+1} r_{B}\right)\right]^{\alpha-\frac{1}{p}} \sup _{B} \frac{\left[\phi\left(\mu\left(2 \times 6^{k+1} B\right)\right)\right]^{\frac{1}{p}}}{[\phi(\mu(8 B))]^{\frac{1}{p}}} \\
& \times\left(\int_{B}\left|b(x)-m_{6^{k+1} B}(b)\right|^{q} \mathrm{~d} \mu(x)\right)^{\frac{1}{q}} \\
& \leq C\|b\|_{\widetilde{\operatorname{RBO}}(\mu)}\|f\|_{L^{p, \phi}(\mu)} \sum_{k=1}^{\infty} \frac{1}{\left[C\left(6^{k+1}\right)\right]^{\frac{1}{p}-\alpha}} \sup _{B} \frac{\left[\phi\left(\mu\left(2 \times 6^{k+1} B\right)\right)\right]^{\frac{1}{p}}}{[\phi(\mu(8 B))]^{\frac{1}{p}}} \\
& +C\|f\|_{L^{p, \phi}(\mu)} \sum_{k=1}^{\infty} \frac{1}{\left[C\left(6^{k+1}\right)\right]^{\frac{1}{p}-\alpha}} \frac{1}{\left[\lambda\left(c_{B, r_{B}}\right)\right]^{\frac{1}{p}-\alpha}} \sup _{B} \frac{\left[\phi\left(\mu\left(2 \times 6^{k+1} B\right)\right)\right]^{\frac{1}{p}}}{[\phi(\mu(8 B))]^{\frac{1}{p}}} \\
& \times\left\{\left(\int_{B}\left|b(x)-m_{\widetilde{B}}(b)\right|^{q} \mathrm{~d} \mu(x)\right)^{\frac{1}{q}}+[\mu(B)]^{\frac{1}{p}-\alpha}\left|m_{\widetilde{B}}(b)-m_{6^{k+1} B}(b)\right|\right\} \\
& \leq C\|b\|_{\overparen{\operatorname{RBMO}}(\mu)}\|f\|_{L^{p, \phi}(\mu)} \sum_{k=1}^{\infty} \frac{1+k}{\left[C\left(6^{k+1}\right)\right]^{\frac{1}{p}-\alpha}} \sup _{B} \frac{\left[\phi\left(\mu\left(2 \times 6^{k+1} B\right)\right)\right]^{\frac{1}{p}}}{[\phi(\mu(8 B))]^{\frac{1}{p}}} \\
& \leq C\|b\|_{\widetilde{\operatorname{RMO}}(\mu)}\|f\|_{L^{p, \phi}(\mu)} \sum_{k=1}^{\infty} \frac{1+k}{\left[C\left(6^{k+1}\right)\right]^{\frac{1}{p}-\alpha}} \sup _{B} \frac{\left[\phi\left(\lambda\left(c_{B}, 2 \times 6^{k+1} r_{B}\right)\right)\right]^{\frac{1}{p}}}{\left[\phi\left(\lambda\left(c_{B}, 6^{k+1} r_{B}\right)\right)\right]^{\frac{1}{p}}} \\
& \times \frac{\left[\phi\left(\lambda\left(c_{B}, 6^{k+1} r_{B}\right)\right)\right]^{\frac{1}{p}}}{\left[\phi\left(\lambda\left(c_{B}, \frac{1}{2} \times 6^{k+1} r_{B}\right)\right)\right]^{\frac{1}{p}}} \times \cdots \times \frac{\left[\phi\left(\lambda\left(c_{B}, r_{B}\right)\right)\right]^{\frac{1}{p}}}{[\phi(\mu(8 B))]^{\frac{1}{p}}} \\
& \leq C\|b\|_{\widehat{\operatorname{RBMO}}(\mu)}\|f\|_{L^{p, \phi}(\mu)} \sum_{k=1}^{\infty} \frac{(1+k) C_{\lambda}^{\frac{k}{p}}}{\left[C\left(6^{k+1}\right)\right]^{\frac{1}{p}-\alpha}} \\
& \leq C\|b\|_{\overparen{\operatorname{RBMO}}(\mu)}\|f\|_{L^{p, \phi}(\mu)} .
\end{aligned}
$$

Hence, the proof of Theorem 6.2 is finished. 


\section{Funding}

This research is supported by the Innovation Capacity Improvement Project for Colleges and Universities of Gansu Province (no. 2020A-010), The Young Teachers' Scientific Research Ability Promotion Project of Northwest Normal University (no. NWNU-LKQN2020-07) and the Doctoral Scientific Research Foundation of Northwest Normal University (no. 6014/0002020203)

\section{Availability of data and materials}

Not applicable.

\section{Competing interests}

There do not exist any competing interests regarding this article.

\section{Authors' contributions}

The author read and approved the final manuscript.

\section{Publisher's Note}

Springer Nature remains neutral with regard to jurisdictional claims in published maps and institutional affiliations.

Received: 13 November 2019 Accepted: 31 July 2020 Published online: 08 August 2020

\section{References}

1. Bui, T.A., Duong, X.T.: Hardy spaces, regularized BMO spaces and the boundedness of Calderón-Zygmund operators on non-homogeneous spaces. J. Geom. Anal. 23(2), 895-932 (2013)

2. Cao, Y., Zhou, Z.: Morrey spaces for nonhomogeneous metric measure spaces. Abstr. Appl. Anal. 2013(4), 205 (2013)

3. Chen, W., Sawyer, E.: A note on commutators of fractional integrals with RBMO $(\mu)$ functions. III. J. Math. 46(4), 1287-1298 (2002)

4. Coifman, R.R., Weiss, G.: Analyse Harmonique Non-commutative Sur Certains Espaces Homogènes. (French) Étude de Certaines Intégrales Singulières. Lecture Notes in Mathematics, vol. 242. Springer, Berlin (1971)

5. Coifman, R.R., Weiss, G.: Extensions of Hardy spaces and their use in analysis. Bull. Am. Math. Soc. 83(4), 569-645 (1977)

6. Fu, X., Yang, D., Yang, D.: The molecular characterization of the Hardy space $H^{1}$ on non-homogeneous metric measure spaces and its application. J. Math. Anal. Appl. 410(1), 1028-1042 (2014)

7. Fu, X., Yang, D., Yuan, W.: Generalized fractional integrals and their commutators over non-homogeneous metric measure spaces. Taiwan. J. Math. 18(2), 509-557 (2014)

8. Hu, G., Lin, H., Yang, D.: Marcinkiewicz integrals with non-doubling measures. Integral Equ. Oper. Theory 58(2), 205-238 (2007)

9. Hytönen, T.: A framework for non-homogeneous analysis on metric spaces, and RBMO space of Tolsa. Publ. Math. 54(2), 485-504 (2010)

10. Hytönen, T., Yang, D., Yang, D.: The Hardy space $H^{1}$ on non-homogeneous metric spaces. Math. Proc. Camb. Philos. Soc. 153(1), 9-31 (2012)

11. Li, H., Lin, H.: Boundedness of Marcinkiewicz integrals on Hardy spaces $H^{p}$ over non-homogeneous metric measure spaces. J. Math. Inequal. 12(2), 347-364 (2018)

12. Lin, H., Wu, S., Yang, D.: Boundedness of certain commutators over non-homoge- neous metric measure spaces. Anal. Math. Phys. 7(2), 187-218 (2017)

13. Lu, G.: Commutators of bilinear $\theta$-type Calderón-Zygmund operators on Morrey spaces over non-homogeneous spaces. Anal. Math. 46(1), 97-118 (2020)

14. Lu, G., Tao, S.: Generalized Morrey spaces over non-homogeneous metric measure spaces. J. Aust. Math. Soc. 103(2), 268-278 (2017)

15. Meng, Y., Yang, D.: Multilinear commutators of Calderón-Zygmund operators on Hardy-type spaces with non-doubling measures. J. Math. Anal. Appl. 317(1), 228-244 (2006)

16. Ri, C., Zhang, Z.: Boundedness of $\theta$-type Calderón-Zygmund operators on non-homogeneous metric measure spaces. Chin. Ann. Math., Ser. B 40(4), 585-598 (2019)

17. Sawano, Y.: Generalized Morrey spaces for non-doubling measures. Nonlinear Differ. Equ. Appl. 15(4), 413-425 (2008)

18. Sawano, Y., Tanaka, H.: Morrey spaces for non-doubling measures. Acta Math. Sin. Engl. Ser. 21(6), 1535-1544 (2005)

19. Tao, S., Lu, G.: Commutators of Marcinkiewicz integrals with $\widehat{\operatorname{RBMO}}(\mu)$ on Morrey spaces. Acta Math. Sinica (Chin. Ser.) 62(2), 269-278 (2019)

20. Tolsa, X.: BMO, $H^{1}$ and Calderón-Zygmund operator for non-doubling measures. Math. Ann. 319(1), 89-149 (2001)

21. Tolsa, X.: Littlewood-Paley theory and the T(1) theorem with non-doubling measures. Adv. Math. 164(1), 57-116 (2001)

22. Wang, M., Ma, S., Lu, G.: Littlewood-Paley $g_{\lambda}^{*}$-function and its commutator on non-homogeneous generalized Morrey spaces. Tokyo J. Math. 41(2), 617-626 (2018)

23. Yang, D., Yang, D., Hu, G.: The Hardy Space $H^{1}$ with Non-doubling Measures and Their Applications. Lecture Notes in Mathematics, vol. 2084. Springer, Berlin (2013) 